Article

\title{
Gauss Map and Its Applications on Ruled Submanifolds in Minkowski Space
}

\author{
Sun Mi Jung and Young Ho Kim * \\ Department of Mathematics, Kyungpook National University, Daegu 41566, Korea; jung3756@knu.ac.kr \\ * Correspondence: yhkim@knu.ac.kr; Tel.: +82-53-950-5888
}

Received: 12 May 2018; Accepted: 11 June 2018; Published: 13 June 2018

\begin{abstract}
We study ruled submanifolds in Minkowski space in regard to the Gauss map satisfying some partial differential equation. As a generalization of usual cylinders, cones and null scrolls in a three-dimensional Minkowski space, a cylinder over a space curve, a product manifold of a right cone and a $k$-plane, a product manifold of a hyperbolic cone and a $k$-plane which look like kinds of cylinders over cones in 3-space, and the generalized B-scroll kind in Minkowski space are characterized with the partial differential equation regarding the Gauss map, where $k$ is a positive integer.
\end{abstract}

Keywords: finite-type immersion; pointwise 1-type Gauss map of the second kind; generalized $B$-scroll kind

\section{Introduction}

According to Nash's imbedding theorem, a Riemannian manifold can be imbedded in a Euclidean space with considerably high codimension. That naturally enables us to study Riemannian manifolds as submanifolds of a Euclidean space. In the late 1970's, the notion of finite-type immersion of Riemannian manifolds into Euclidean space was introduced, which is a generalization of the so-called eigenvalue problem of the immersion [1]: An isometric immersion of $x$ of a Riemannian manifold $M$ into a Euclidean space $\mathbb{E}^{m}$ is said to be of finite-type if it can be expressed as

$$
x=x_{0}+x_{1}+\cdots+x_{k}
$$

for some positive integer $k$, where $x_{0}$ is a constant vector and $\Delta x_{i}=\lambda_{i} x_{i}$ for some $\lambda_{i} \in \mathbb{R}, i=1, \ldots, k$. Here, $\Delta$ denotes the Laplace operator defined on $M$. If $\lambda_{1}, \ldots, \lambda_{k}$ are mutually different, $M$ is said to be of $k$-type. We may assume that a finite-type immersion of $x$ of a Riemannian manifold into a Euclidean space is of $k$-type for some non-negative integer $k$.

Let $\mathbb{E}_{s}^{m}$ be an $m$-dimensional pseudo-Euclidean space of signature $(m-s, s)$. The notion of finite-type immersion was extended to that of submanifolds in pseudo-Euclidean space $\mathbb{E}_{s}^{m}$ and to that of smooth maps defined on submanifolds of Euclidean space $\mathbb{E}^{m}$ or pseudo-Euclidean space $\mathbb{E}_{s}^{m}$. In particular, the study of finite-type immersions and finite-type Gauss map of submanifolds in the Minkowski $m$-space $\mathbb{E}_{1}^{m}$ denoted by $\mathbb{L}^{m}$ has been made extensively ([2-15]).

On the other hand, the Gauss map of some nice surfaces in the three-dimensional Euclidean space $\mathbb{E}^{3}$ has an interesting property regarding the Laplacian. The helicoid in $\mathbb{E}^{3}$ parameterized by

$$
x(u, v)=(u \cos v, u \sin v, a v), a \neq 0
$$

has the Gauss map G satisfying

$$
\Delta G=\frac{2 a^{2}}{\left(a^{2}+u^{2}\right)^{2}} G
$$

The Gauss map of the right (or circular) cone in $\mathbb{E}^{3}$ with parametrization 


$$
x(u, v)=(u \cos v, u \sin v, a u), a \geq 0
$$

satisfies

$$
\Delta G=\frac{1}{u}\left(G+\left(0,0, \frac{1}{\sqrt{1+a^{2}}}\right)\right)
$$

(cf. $[16,17])$. The Gauss map of those surfaces is similar to of 1-type, but obviously not of 1-type in the usual sense. We need to know what other manifolds have such a property. Based on these examples, the following definition was introduced.

Definition 1 ([18]). An oriented $n$-dimensional submanifold $M$ of the Euclidean space $\mathbb{E}^{m}$ or the pseudoEuclidean space $\mathbb{E}_{s}^{m}$ is said to have pointwise 1-type Gauss map or the Gauss map is of pointwise 1-type if it satisfies

$$
\Delta G=f(G+C),
$$

where $f$ is a non-zero smooth function on $M$ and $C$ a constant vector in the ambient space. In particular, if $C$ is zero, the Gauss map $G$ is said to be of pointwise 1-type of the first kind. Otherwise, it is said to be of the second kind ([19-24]).

The notion of ruled submanifold is a concept of great interest in the Riemannian geometry, which has been investigated by many authors. Several results involving ruled submanifolds in manifolds equipped with remarkable geometric structures were recently obtained in [25-28].

In $[19,20]$, the authors of the present paper et al. studied ruled submanifolds in the Euclidean space $\mathbb{E}^{m}$ with pointwise 1-type Gauss map and proved that the ruled submanifold $M$ in the Euclidean space $\mathbb{E}^{m}$ is minimal if and only if the Gauss map $G$ of $M$ is of pointwise 1-type Gauss map of the first kind. Further, we showed that the only non-cylindrical ruled submanifold $M$ in the Euclidean space $\mathbb{E}^{m}$ with pointwise 1-type Gauss map of the second kind is the generalized right cone.

In [29], the authors of the present paper and et al. investigated the ruled submanifolds in the Lorentz-Minkowski $m$-space $\mathbb{L}^{m}$ with pointwise 1-type Gauss map of the first kind and then established the equivalent conditions for the minimality of the ruled submanifold in the Lorentz-Minkowski $m$-space $\mathbb{L}^{m}$ by means of the Gauss map.

In this paper, we will study ruled submanifolds in $\mathbb{L}^{m}$ with pointwise 1-type Gauss map of the second kind and thereby complete the classification of the ruled submanifolds in $\mathbb{L}^{m}$ with pointwise 1-type Gauss map.

\section{Preliminaries}

A curve in $\mathbb{E}_{s}^{m}$ is said to be space-like, time-like or null if its tangent vector field is space-like, time-like or null, respectively.

Let $x: M \rightarrow \mathbb{E}_{s}^{m}$ be an isometric immersion of an $n$-dimensional pseudo-Riemannian manifold $M$ into $\mathbb{E}_{s}^{m}$. Throughout the present paper, a submanifold in $\mathbb{E}_{s}^{m}$ always means pseudo-Riemannian, in other words, each tangent space of the submanifold in $\mathbb{E}_{s}^{m}$ is non-degenerate.

Let $\left(x_{1}, x_{2}, \ldots, x_{n}\right)$ be a local coordinate system of $M$ in $\mathbb{E}_{s}^{m}$. For the components $g_{i j}$ of the pseudo-Riemannian metric $\langle\cdot, \cdot\rangle$ on $M$ induced from that of $\mathbb{E}_{s}^{m}$, we denote by $\left(g^{i j}\right)$ (respectively, $\mathcal{G}$ ) the inverse matrix (respectively, the determinant) of the matrix $\left(g_{i j}\right)$ of the components of the induced metric $\langle\cdot, \cdot\rangle$. Then, the Laplacian $\Delta$ defined on $M$ is given by

$$
\Delta=-\frac{1}{\sqrt{|\mathcal{G}|}} \sum_{i, j} \frac{\partial}{\partial x_{i}}\left(\sqrt{|\mathcal{G}|} g^{i j} \frac{\partial}{\partial x_{j}}\right) .
$$

We now define the Gauss map $G$ on $M$. Consider the map $G: M \rightarrow G(n, m)$ of a point $p$ of $M$ mapped to an oriented tangent space at $p$, where $G(n, m)$ is the Grassmannian manifold consisting of all oriented $n$-planes passing through the origin. Roughly speaking it can be achieved by parallel 
displacement of the oriented tangent space at $p$ to the origin of $\mathbb{L}^{m}$. By an isomorphism, $G(n, m)$ can be identified with $G(m-n, m)$ in a natural manner. Let us express the Gauss map rigorously. Choose an adapted local orthonormal frame $\left\{e_{1}, e_{2}, \ldots, e_{m}\right\}$ in $\mathbb{E}_{s}^{m}$ such that $e_{1}, e_{2}, \ldots, e_{n}$ are tangent to $M$ and $e_{n+1}, e_{n+2}, \ldots, e_{m}$ normal to $M$. Define the map $G: M \rightarrow G(n, m) \subset \mathbb{R}^{N}\left(N={ }_{m} C_{n}\right)$, $G(p)=\left(e_{1} \wedge e_{2} \wedge \cdots \wedge e_{n}\right)(p)$.

An indefinite scalar product $\ll \cdot, \cdot \gg$ on $G(n, m) \subset \mathbb{R}^{N}$ is defined by

$$
\ll e_{i_{1}} \wedge \cdots \wedge e_{i_{n}}, e_{j_{1}} \wedge \cdots \wedge e_{j_{n}} \gg=\operatorname{det}\left(\left\langle e_{i_{l}}, e_{j_{k}}\right\rangle\right) .
$$

Then, $\left\{e_{i_{1}} \wedge e_{i_{2}} \wedge \cdots \wedge e_{i_{n}} \mid 1 \leq i_{1}<\cdots<i_{n} \leq m\right\}$ is an orthonormal basis of $\mathbb{E}_{k}^{N}$ for some positive integer $k$.

Now, let us recall the notion of a ruled submanifold $M$ in $\mathbb{L}^{m}$ ([7-10]). A non-degenerate $(r+$ 1)-dimensional submanifold $M$ in $\mathbb{L}^{m}$ is called a ruled submanifold if $M$ is foliated by $r$-dimensional totally geodesic submanifolds $E(s, r)$ of $\mathbb{L}^{m}$ along a regular curve $\alpha=\alpha(s)$ on $M$ defined on an open interval $I$. Thus, a parametrization of a ruled submanifold $M$ in $\mathbb{L}^{m}$ can be given by

$$
x=x\left(s, t_{1}, t_{2}, \ldots, t_{r}\right)=\alpha(s)+\sum_{i=1}^{r} t_{i} e_{i}(s), s \in I, t_{i} \in I_{i}
$$

where $I_{i}$ 's are some open intervals for $i=1,2, \ldots, r$. Without loss of generality, we may assume that $0 \in I_{i}$ for all $i=1,2, \ldots, r$. For each $s, E(s, r)$ is open in $\operatorname{Span}\left\{e_{1}(s), e_{2}(s), \ldots, e_{r}(s)\right\}$, which is the linear span of linearly independent vector fields $e_{1}(s), e_{2}(s), \ldots, e_{r}(s)$ along the curve $\alpha$. Here, we assume that $E(s, r)$ are either non-degenerate or degenerate for all $s$ along $\alpha$. We call $E(s, r)$ the rulings and $\alpha$ the base curve of the ruled submanifold $M$. In particular, the ruled submanifold $M$ is said to be cylindrical if $E(s, r)$ are parallel along $\alpha$, or non-cylindrical otherwise.

Remark $1([7,8])$. (1) If the rulings of $M$ are non-degenerate, then the base curve $\alpha$ can be chosen to be orthogonal to the rulings as follows: Let $V$ be a unit vector field on $M$ which is orthogonal to the rulings. Then $\alpha$ can be taken as an integral curve of $V$.

(2) If the rulings are degenerate, we can choose a null base curve which is transversal to the rulings: Let $V$ be a null vector field on $M$ which is not tangent to the rulings. An integral curve of $V$ can be the base curve.

By solving a system of ordinary differential equations similarly set up relative to a frame along a curve in $\mathbb{L}^{m}$ as given in [30], we have

Lemma 1 ([8]). Let $V(s)$ be a smooth l-dimensional non-degenerate distribution in the Minkowski m-space $\mathbb{L}^{m}$ along a curve $\alpha=\alpha(s)$, where $l \geq 2$ and $m \geq 3$. Then, we can choose orthonormal vector fields $e_{1}(s), \ldots, e_{m-l}(s)$ along $\alpha$ which generate the orthogonal complement $V^{\perp}(s)$ satisfying $e_{i}^{\prime}(s) \in V(s)$ for $1 \leq i \leq m-l$.

\section{Characterization of Cylinders over Spatial Base Curves}

Let $M$ be an $(r+1)$-dimensional ruled submanifold in $\mathbb{L}^{m}$ with non-degenerate rulings. Then, by Remark 1 , the base curve $\alpha$ can be chosen to be orthogonal to the rulings. Without loss of generality, we may assume that $\alpha$ is a unit speed curve, that is, $\left\langle\alpha^{\prime}(s), \alpha^{\prime}(s)\right\rangle=\varepsilon(= \pm 1)$. From now on, the prime ' denotes $d / d s$ unless otherwise stated. By Lemma 1, we may choose orthonormal vector fields $e_{1}(s), \ldots, e_{r}(s)$ along $\alpha$ satisfying

$$
\left\langle\alpha^{\prime}(s), e_{i}(s)\right\rangle=0,\left\langle e_{i}^{\prime}(s), e_{j}(s)\right\rangle=0, i, j=1,2, \ldots, r .
$$

A parametrization of $M$ is given by 


$$
x=x\left(s, t_{1}, t_{2}, \ldots, t_{r}\right)=\alpha(s)+\sum_{i=1}^{r} t_{i} e_{i}(s) .
$$

In this section, we always assume that the parametrization (3) satisfies condition (2). Then, the Gauss map $G$ of $M$ is given by

$$
G=\frac{1}{\left\|x_{s}\right\|} x_{s} \wedge x_{t_{1}} \wedge \cdots \wedge x_{t_{r}}
$$

or, equivalently

$$
G=\frac{1}{|q|^{1 / 2}}\left(\Phi+\sum_{i=1}^{r} t_{i} \Psi_{i}\right)
$$

where $q, \Phi$ and $\Psi_{i}$ are the function and the vectors respectively, defined by

$$
q=\left\langle x_{s}, x_{s}\right\rangle, \quad \Phi=\alpha^{\prime} \wedge e_{1} \wedge \cdots \wedge e_{r} \quad \text { and } \quad \Psi_{i}=e_{i}^{\prime} \wedge e_{1} \wedge \cdots \wedge e_{r} .
$$

First, we consider the case of cylindrical ruled submanifolds that are one of two typical types of ruled submanifolds, which are cylindrical or non-cylindrical. Before discussing cylindrical ruled submanifolds, we cite the following lemma.

Lemma 2 ([29]). Suppose that a unit speed curve $\alpha(s)$ in the $m$-dimensional Minkowski space $\mathbb{L}^{m}$ defined on an open interval I satisfies

$$
\alpha^{\prime \prime \prime}(s)=g(s)\left(\alpha^{\prime}(s)+C\right),
$$

where $g$ is a function of the parameter s and $C$ a constant vector in $\mathbb{L}^{m}$. Then, the curve $\alpha$ lies in a 3-dimensional affine space in $\mathbb{L}^{m}$. In particular, if the constant vector $C$ is zero, we see that $\alpha$ is a plane curve.

We now prove that if an $(r+1)$-dimensional cylindrical ruled submanifold $M$ in $\mathbb{L}^{m}$ has pointwise 1-type Gauss map of the second kind satisfying (1), then it is part of a $(r+1)$-plane or a cylinder over a curve in 3-dimensional affine space.

Let $M$ be a cylindrical $(r+1)$-dimensional ruled submanifold in $\mathbb{L}^{m}$ generated by non-degenerate rulings which is parameterized by (3). Without loss of generality, we may assume that $e_{1}, e_{2}, \ldots, e_{r}$ generating the rulings are constant vectors.

The Laplacian $\Delta$ of $M$ is then naturally expressed by

$$
\Delta=-\varepsilon \frac{\partial^{2}}{\partial s^{2}}-\sum_{i=1}^{r} \varepsilon_{i} \frac{\partial^{2}}{\partial t_{i}^{2}}
$$

where $\varepsilon_{i}=\left\langle e_{i}(s), e_{i}(s)\right\rangle= \pm 1$ and the Gauss map $G$ of $M$ is given by

$$
G=\varepsilon \alpha^{\prime} \wedge e_{1} \wedge \cdots \wedge e_{r}=\varepsilon \Phi .
$$

We now suppose that the Gauss map $G$ is of pointwise 1-type of the second kind, that is, $\Delta G=f(G+\mathbf{C})$ for some non-zero smooth function $f$ and some non-zero constant vector $\mathbf{C}$. Then, the equation $\Delta G=f(G+\mathbf{C})$ is written as

$$
-\varepsilon \Phi^{\prime \prime}=f(\varepsilon \Phi+\mathbf{C}) .
$$

From Equation (6), we see that $f$ is a function of $s$. We may assume that $f$ is non-zero on the open interval $I=\operatorname{dom}(\alpha)$. Then, differentiation of Equation (6) with respect to $s$ gives

$$
\frac{\varepsilon f^{\prime}}{f^{2}} \Phi^{\prime \prime}-\frac{\varepsilon}{f} \Phi^{\prime \prime \prime}-\varepsilon \Phi^{\prime}=\mathbf{0},
$$


or, equivalently

$$
\frac{f^{\prime}}{f^{2}} \alpha^{\prime \prime \prime}-\frac{1}{f} \alpha^{(4)}-\alpha^{\prime \prime}=\mathbf{0}
$$

which implies that $-\frac{1}{f} \alpha^{\prime \prime \prime}-\alpha^{\prime}=\mathbf{D}$ for some constant vector $\mathbf{D}$, where $\mathbf{0}$ denotes zero vector. Namely, if we denote by $\Delta^{\prime}$ the Laplacian of $\alpha$, we have

$$
\Delta^{\prime} \alpha^{\prime}=-\alpha^{\prime \prime \prime}=f\left(\alpha^{\prime}+\mathbf{D}\right) .
$$

According to Lemma 2, we see that the curve $\alpha$ lies in a 3-dimensional affine space in $\mathbb{L}^{m}$.

If a cylindrical ruled submanifold $M$ is part of an $(r+1)$-plane or a cylinder over a 3-dimensional affine space satisfying (8), it is obvious that the Gauss map $G$ is of pointwise 1-type of the second kind. Thus, we have

Theorem 1. Let $M$ be an $(r+1)$-dimensional cylindrical ruled submanifold of $\mathbb{L}^{m}$. Then, $M$ has pointwise 1-type Gauss map of the second kind if and only if $M$ is part of an $(r+1)$-dimensional plane or a cylinder over a curve in a 3-dimensional affine space in $\mathbb{L}^{m}$ satisfying (8).

Next, we consider the case that non-cylindrical ruled submanifolds have pointwise 1-type Gauss map of the second kind. Let $M$ be an $(r+1)$-dimensional non-cylindrical ruled submanifold parameterized by (3) in $\mathbb{L}^{m}$. Then, we have

$$
x_{s}=\alpha^{\prime}(s)+\sum_{j=1}^{r} t_{j} e_{j}^{\prime}(s), \quad x_{t_{i}}=e_{i}(s)
$$

for $i=1,2, \ldots, r$. The function $q$ defined in the beginning of this section is given by

$$
q=\left\langle x_{s}, x_{s}\right\rangle=\varepsilon+\sum_{i=1}^{r} 2 u_{i} t_{i}+\sum_{i, j=1}^{r} w_{i j} t_{i} t_{j}
$$

where $u_{i}(s)=\left\langle\alpha^{\prime}, e_{i}^{\prime}\right\rangle$ and $w_{i j}(s)=\left\langle e_{i}^{\prime}, e_{j}^{\prime}\right\rangle$ for $i, j=1, \ldots, r$. Note that $q$ is a polynomial in $t=\left(t_{1}, \ldots, t_{r}\right)$ with functions in $s$ as coefficients.

From now on, for a polynomial $F(t)$ in $t=\left(t_{1}, t_{2}, \ldots, t_{r}\right), \operatorname{deg} F(t)$ denotes the degree of $F(t)$ in $t=\left(t_{1}, t_{2}, \ldots, t_{r}\right)$ unless otherwise stated.

If we adapt the proof of Proposition 3.3 of [19] to the case of a non-cylindrical ruled submanifold in the Minkowski $m$-space $\mathbb{L}^{m}$, we may assume that the generator vector fields $e_{1}, e_{2}, \ldots, e_{r}$ of the rulings of $M$ satisfy

$$
e_{j}^{\prime} \neq \mathbf{0}
$$

on the domain $I$ of $\alpha$ for all $j=1,2, \ldots, r$ if $M$ has pointwise 1-type Gauss map of the second kind. Then, we get the components of the metric $\langle\cdot, \cdot \cdot\rangle$ on $M$

$$
g_{11}=q, \quad g_{1 j}=0 \quad \text { and } \quad g_{i j}=\varepsilon_{i} \delta_{i j}
$$

for $i, j=2,3, \ldots, r+1$.

It is enough for us to consider the case of $q>0$. Accordingly, Equation (9) gives $\varepsilon=1$. By definition, we have the Laplacian of the form

$$
\Delta=\frac{1}{2 q^{2}} \frac{\partial q}{\partial s} \frac{\partial}{\partial s}-\frac{1}{q} \frac{\partial^{2}}{\partial s^{2}}-\frac{1}{2 q} \sum_{i=1}^{r} \varepsilon_{i} \frac{\partial q}{\partial t_{i}} \frac{\partial}{\partial t_{i}}-\sum_{i=1}^{r} \varepsilon_{i} \frac{\partial^{2}}{\partial t_{i}^{2}} .
$$


First, we suppose that $e_{1}^{\prime}, e_{2}^{\prime}, \ldots, e_{r}^{\prime}$ are non-null. Then, using the Formula (10), $\Delta G=f(G+\mathbf{C})$ can be expressed as

$$
\begin{array}{r}
\left(\frac{\partial q}{\partial s}\right)^{2}\left(\Phi+\sum_{j=1}^{r} \Psi_{j} t_{j}\right)-\frac{3}{2} q \frac{\partial q}{\partial s}\left(\Phi^{\prime}+\sum_{j=1}^{r} \Psi_{j}^{\prime} t_{j}\right)-\frac{1}{2} q \frac{\partial^{2} q}{\partial s^{2}}\left(\Phi+\sum_{j=1}^{r} \Psi_{j} t_{j}\right) \\
+q^{2}\left(\Phi^{\prime \prime}+\sum_{j=1}^{r} \Psi_{j}^{\prime \prime} t_{j}\right)+\frac{1}{2} q \sum_{i=1}^{r} \varepsilon_{i}\left(\frac{\partial q}{\partial t_{i}}\right)^{2}\left(\Phi+\sum_{j=1}^{r} \Psi_{j} t_{j}\right)-\frac{1}{2} q^{2} \sum_{i=1}^{r} \varepsilon_{i} \frac{\partial q}{\partial t_{i}} \Psi_{i} \\
-\frac{1}{2} q^{2} \sum_{i=1}^{r} \varepsilon_{i} \frac{\partial^{2} q}{\partial t_{i}^{2}}\left(\Phi+\sum_{j=1}^{r} \Psi_{j} t_{j}\right)+f\left\{q^{3}\left(\Phi+\sum_{j=1}^{r} \Psi_{j} t_{j}\right)+q^{\frac{7}{2}} \mathbf{C}\right\}=\mathbf{0} .
\end{array}
$$

If we use the indefinite scalar product $\ll \cdot, \cdot \gg$ on $G(r+1, m)$, we have

$$
\begin{aligned}
& \ll \Phi, \Phi \gg=\tilde{\varepsilon}, \quad \ll \Phi, \Phi^{\prime} \gg=0, \\
& \ll \Phi, \Phi^{\prime \prime} \gg=-\tilde{\varepsilon} \mu+2 \sum_{k=1}^{r} \tilde{\varepsilon} \varepsilon_{k} u_{k}^{2}-\sum_{k=1}^{r} \tilde{\varepsilon} \varepsilon_{k} w_{k k}, \\
& \ll \Phi, \Psi_{i} \gg=\tilde{\varepsilon} u_{i}, \quad \ll \Phi, \Psi_{i}^{\prime} \gg=\tilde{\varepsilon} p_{i}, \\
& \ll \Phi, \Psi_{i}^{\prime \prime} \gg=\tilde{\varepsilon} y_{i}+2 \sum_{k=1}^{r} \tilde{\varepsilon} \varepsilon_{k} u_{k} w_{i k}-\sum_{k=1}^{r} \tilde{\varepsilon} \varepsilon_{k} u_{i} w_{k k}, \\
& \ll \Psi_{i}, \Phi^{\prime} \gg=\tilde{\varepsilon} z_{i}, \quad \ll \Psi_{i}, \Psi_{j} \gg=\tilde{\varepsilon} w_{i j}, \quad \ll \Psi_{i}, \Psi_{j}^{\prime} \gg=\tilde{\varepsilon} \tilde{\xi}_{i j},
\end{aligned}
$$

where we have put

$$
\tilde{\varepsilon}=\varepsilon_{1} \cdots \varepsilon_{r}, \mu=\left\langle\alpha^{\prime \prime}, \alpha^{\prime \prime}\right\rangle, p_{i}=\left\langle\alpha^{\prime}, e_{i}^{\prime \prime}\right\rangle, y_{i}=\left\langle\alpha^{\prime}, e_{i}^{\prime \prime \prime}\right\rangle, z_{i}=\left\langle\alpha^{\prime \prime}, e_{i}^{\prime}\right\rangle, \xi_{i j}=\left\langle e_{i}^{\prime}, e_{j}^{\prime \prime}\right\rangle .
$$

Then, we get

$$
u_{i}^{\prime}(s)=p_{i}(s)+z_{i}(s) \text { and } \quad w_{i j}^{\prime}=\xi_{i j}+\xi_{j i} .
$$

By taking the indefinite scalar product with the vector $\Phi$ to both sides of (11), we obtain

$$
\begin{array}{r}
\left(\frac{\partial q}{\partial s}\right)^{2}\left(1+\sum_{j=1}^{r} u_{j} t_{j}\right)-\frac{3}{2} q \frac{\partial q}{\partial s}\left(\sum_{j=1}^{r} p_{j} t_{j}\right)-\frac{1}{2} q \frac{\partial^{2} q}{\partial s^{2}}\left(1+\sum_{j=1}^{r} u_{j} t_{j}\right) \\
+q^{2}\left(\tilde{\varepsilon} \phi+\sum_{j=1}^{r} \tilde{\varepsilon} \varphi_{j} t_{j}\right)+\frac{1}{2} q \sum_{i=1}^{r} \varepsilon_{i}\left(\frac{\partial q}{\partial t_{i}}\right)^{2}\left(1+\sum_{j=1}^{r} u_{j} t_{j}\right)-\frac{1}{2} q^{2} \sum_{i=1}^{r} \varepsilon_{i} \frac{\partial q}{\partial t_{i}} u_{i} \\
-\frac{1}{2} q^{2} \sum_{i=1}^{r} \varepsilon_{i} \frac{\partial^{2} q}{\partial t_{i}^{2}}\left(1+\sum_{j=1}^{r} u_{j} t_{j}\right)+f\left\{q^{3}\left(1+\sum_{j=1}^{r} u_{j} t_{j}\right)+q^{\frac{7}{2}} \gamma(s)\right\}=0,
\end{array}
$$

where we have put

$$
\gamma(s)=\ll \mathbf{C}, \Phi(s) \gg, \phi=\ll \Phi, \Phi^{\prime \prime} \gg \quad \text { and } \quad \varphi_{i}=\ll \Phi, \Psi_{i}^{\prime \prime} \gg .
$$

Let $\bar{e}_{r+1}, \bar{e}_{r+2}, \ldots, \bar{e}_{m-1}$ be the orthonormal vector fields which are normal to $M$ along $\alpha$. If we apply Lemma 1 to the normal space $T_{\alpha(s)}^{\perp} M$ of $M$, then there exists an orthonormal frame $\left\{e_{a}\right\}_{a=r+1}^{m-1}$ of the normal space $T_{\alpha(s)}^{\perp} M$ satisfying

$$
\left\langle e_{a}^{\prime}(s), e_{b}(s)\right\rangle=0
$$

for all $a, b=r+1, \ldots, m-1$. Then we can put

$$
e_{j}^{\prime}=u_{j} \alpha^{\prime}+\sum_{a=r+1}^{m-1} \varepsilon_{a} \lambda_{a}^{j} e_{a}
$$


where $\varepsilon_{a}=\left\langle e_{a}, e_{a}\right\rangle= \pm 1$ and $\lambda_{a}^{j}(s)=\left\langle e_{j}^{\prime}, e_{a}\right\rangle$ for $a=r+1, \ldots, m-1$. From (16), we get

$$
\Psi_{j}=u_{j} \Phi+\sum_{a=r+1}^{m-1} \varepsilon_{a} \lambda_{a}^{j} \xi_{a}
$$

where $\xi_{a}=e_{a} \wedge e_{1} \wedge e_{2} \wedge \cdots \wedge e_{r}$ for $a=r+1, \ldots, m-1$. And, we may put

$$
\alpha^{\prime \prime}=-\sum_{i=1}^{r} \varepsilon_{i} u_{i} e_{i}-\sum_{a=r+1}^{m-1} \varepsilon_{a} u_{a} e_{a}
$$

where $u_{a}(s)=\left\langle\alpha^{\prime}, e_{a}^{\prime}\right\rangle$ for all $a=r+1, \ldots, m-1$.

Suppose that $M$ is not an $(r+1)$-plane, that is, $G \neq-\mathbf{C}$. To deal with (13), we consider the subset

$$
M_{1}=\left\{p \in M \mid q^{3}\left(1+\sum_{j=1}^{r} u_{j} t_{j}\right)+q^{\frac{7}{2}} \gamma(s)=0\right\} .
$$

Without loss of generality, we may assume that $f \neq 0$ on $M_{1}$. Then, on $M_{1}$,

$$
\left(1+\sum_{j=1}^{r} u_{j} t_{j}\right)+\sqrt{q} \gamma(s)=0, \quad \text { or, } \quad\left(1+\sum_{j=1}^{r} u_{j} t_{j}\right)^{2}=q \gamma^{2}(s) .
$$

By (9) and $\varepsilon=1$, we see that $\gamma^{2}(s)=1$ and hence

$$
q=\left(1+\sum_{j=1}^{r} u_{j} t_{j}\right)^{2}
$$

which implies that

$$
M_{1}=\{p \in M \mid 1+\gamma(s)=0\} .
$$

Also, it follows from (16) that on $M_{1}$,

$$
\sum_{a=r+1}^{m-1} \varepsilon_{a} \lambda_{a}^{k} \lambda_{a}^{j}=0
$$

for all $j, k=1, \ldots, r$.

Lemma 3. Let $M$ be an $(r+1)$-dimensional non-cylindrical and non-planar ruled submanifold parameterized by (3) in $\mathbb{L}^{m}$. Let $e_{1}, e_{2}, \ldots, e_{r}$ be the orthonormal generators of the rulings along the base curve $\alpha$ such that $e_{j}^{\prime}$ are non-null for all $j=1,2, \ldots, r$. If the Gauss map $G$ of $M$ satisfies $\Delta G=f(G+C)$ for some non-zero function $f$ and non-zero constant vector $\boldsymbol{C}$, then

$$
\gamma(s)=\ll C, \Phi(s) \gg \neq-1
$$

on $\{p \in M \mid f \neq 0\}$.

Proof. Let $\tilde{I}_{1}=\{s \in I \mid 1+\gamma(s)=0\}$. We suppose that the interior $\operatorname{Int}\left(\tilde{I}_{1}\right)$ of $\tilde{I}_{1}$ is non-empty. If we put 


$$
\begin{aligned}
P(t)= & \left(\frac{\partial q}{\partial s}\right)^{2}\left(1+\sum_{j=1}^{r} u_{j} t_{j}\right)-\frac{3}{2} q \frac{\partial q}{\partial s}\left(\sum_{j=1}^{r} p_{j} t_{j}\right)-\frac{1}{2} q \frac{\partial^{2} q}{\partial s^{2}}\left(1+\sum_{j=1}^{r} u_{j} t_{j}\right) \\
& +q^{2}\left(\tilde{\varepsilon} \phi+\sum_{j=1}^{r} \tilde{\varepsilon} \varphi_{j} t_{j}\right)+\frac{1}{2} q \sum_{i=1}^{r} \varepsilon_{i}\left(\frac{\partial q}{\partial t_{i}}\right)^{2}\left(1+\sum_{j=1}^{r} u_{j} t_{j}\right) \\
& -\frac{1}{2} q^{2} \sum_{i=1}^{r} \varepsilon_{i} \frac{\partial q}{\partial t_{i}} \varepsilon u_{i}-\frac{1}{2} q^{2} \sum_{i=1}^{r} \varepsilon_{i} \frac{\partial^{2} q}{\partial t_{i}^{2}}\left(1+\sum_{j=1}^{r} u_{j} t_{j}\right),
\end{aligned}
$$

Equation (13) tells us that $P(t) \equiv 0$ on $\operatorname{Int}\left(\tilde{I}_{1}\right)$. Using $q=\left(1+\sum_{j} u_{j} t_{j}\right)^{2}$ on $\operatorname{Int}\left(\tilde{I}_{1}\right)$, equation $P(t)=0$ yields that

$$
3\left(\sum_{j=1}^{r} u_{j}^{\prime} t_{j}\right)\left(\sum_{j=1}^{r}\left(u_{j}^{\prime}-p_{j}\right) t_{j}\right)+\left(1+\sum_{j=1}^{r} u_{j} t_{j}\right)\left(\tilde{\varepsilon} \phi+\sum_{j=1}^{r}\left(\tilde{\varepsilon} \varphi_{j}-u_{j}^{\prime \prime}\right) t_{j}\right)=0
$$

which provides

$$
\phi=0, \quad \tilde{\varepsilon} \varphi_{j}=u_{j}^{\prime \prime} \quad \text { and } \quad u_{j}^{\prime}\left(u_{j}^{\prime}-p_{j}\right)=0
$$

as the coefficients of terms containing $t_{j}^{0}, t_{j}^{1}$ and $t_{j}^{2}$, respectively, for $j=1, \ldots, r$.

Now, we will proceed with the proof according to the following steps.

Step 1. $u_{j}^{\prime}=0$ on $\operatorname{Int}\left(\tilde{I}_{1}\right)$.

We suppose that $u_{j}^{\prime} \neq 0$ at some point in $\operatorname{Int}\left(\tilde{I}_{1}\right)$. Then, $u_{j}^{\prime}=p_{j}$ in (21) and hence

$$
z_{j}=\left\langle\alpha^{\prime \prime}, e_{j}^{\prime}\right\rangle=\sum_{a=r+1}^{m-1} \varepsilon_{a} u_{a} \lambda_{a}^{j}=0
$$

because of (12), (16) and (18). Since $\phi=\ll \Phi, \Phi^{\prime \prime} \gg=-\ll \Phi^{\prime}, \Phi^{\prime} \gg$ and $\ll \Phi^{\prime}, \Psi_{j} \gg=\tilde{\varepsilon} z_{j}$,

$$
\ll \Phi^{\prime}, \Phi \gg=\ll \Phi^{\prime}, \Phi^{\prime} \gg=\ll \Phi^{\prime}, \Phi^{\prime \prime} \gg=\ll \Phi^{\prime}, \Psi_{j} \gg=0 .
$$

Now, we suppose that $\gamma^{\prime}\left(s_{0}\right) \neq 0$ for some $s_{0} \in \operatorname{Int} \tilde{I}_{1}$. Then, at $s_{0}$ Equation (11) is rewritten as

$$
\begin{aligned}
& 3\left(\sum_{j=1}^{r} u_{j}^{\prime} t_{j}\right)^{2}\left(\Phi+\sum_{j=1}^{r} \Psi_{j} t_{j}\right)-3\left(1+\sum_{j=1}^{r} u_{j} t_{j}\right)\left(\sum_{j=1}^{r} u_{j}^{\prime} t_{j}\right)\left(\Phi^{\prime}+\sum_{j=1}^{r} \Psi_{j}^{\prime} t_{j}\right) \\
& -\left(1+\sum_{j=1}^{r} u_{j} t_{j}\right)\left(\sum_{j=1}^{r} u_{j}^{\prime \prime} t_{j}\right)\left(\Phi+\sum_{j=1}^{r} \Psi_{j} t_{j}\right)+\left(1+\sum_{j=1}^{r} u_{j} t_{j}\right)^{2}\left(\Phi^{\prime \prime}+\sum_{j=1}^{r} \Psi_{j}^{\prime \prime} t_{j}\right) \\
& +\left(1+\sum_{j=1}^{r} u_{j} t_{j}\right)^{2}\left(\sum_{i=1}^{r} \varepsilon_{i} u_{i}^{2}\right)\left(\Phi+\sum_{j=1}^{r} \Psi_{j} t_{j}\right)-\left(1+\sum_{j=1}^{r} u_{j} t_{j}\right)^{3}\left(\sum_{i=1}^{r} \varepsilon_{i} u_{i} \Psi_{i}\right) \\
& +f\left(1+\sum_{j=1}^{r} u_{j} t_{j}\right)^{4}\left\{\left(\Phi+\sum_{j=1}^{r} \Psi_{j} t_{j}\right)+\left(1+\sum_{j=1}^{r} u_{j} t_{j}\right) \mathbf{C}\right\}=\mathbf{0}
\end{aligned}
$$

and taking the indefinite scalar product with $\Phi^{\prime}$ to (24) gives us the following

$$
f=\frac{3\left(\sum_{j} u_{j}^{\prime} t_{j}\right)\left(\sum_{j} \eta_{j} t_{j}\right)-\left(1+\sum_{j} u_{j} t_{j}\right)\left(\sum_{j} \vartheta_{j} t_{j}\right)}{\gamma^{\prime}\left(1+\sum_{j} u_{j} t_{j}\right)^{4}},
$$

where we have put

$$
\eta_{j}(s)=\ll \Phi^{\prime}, \Psi_{j}^{\prime} \gg \quad \text { and } \quad \vartheta_{j}(s)=\ll \Phi^{\prime}, \Psi_{j}^{\prime \prime} \gg
$$


Substituting (25) into (24) and then considering the constant terms of the equation obtained in such a way, we get

$$
\Phi^{\prime \prime}+\left(\sum_{i=1}^{r} \varepsilon_{i} u_{i}^{2}\right) \Phi-\left(\sum_{i=1}^{r} \varepsilon_{i} u_{i} \Psi_{i}\right)=\mathbf{0} .
$$

By straightforward computation, it follows from (19), (21) and (22) that at $s_{0}$

$$
\Phi^{\prime \prime}=\sum_{a=r+1}^{m-1} \varepsilon_{a}\left(-u_{a}^{\prime}+\sum_{k=1}^{r} \varepsilon_{k} u_{k} \lambda_{a}^{k}\right) \xi_{a}+\sum_{k=1}^{r} \sum_{a=r+1}^{m-1} \varepsilon_{a}\left(u_{k} u_{a}+\left(\lambda_{a}^{k}\right)^{\prime}\right) \Gamma_{a}^{k}
$$

where we have put

$$
\Gamma_{a}^{k}=\alpha^{\prime} \wedge e_{1} \wedge \cdots \wedge e_{k-1} \wedge e_{a} \wedge e_{k+1} \wedge \cdots \wedge e_{r} .
$$

Combining (17), (26) and (27), we can obtain

$$
u_{a}^{\prime}=0 \quad \text { and } \quad\left(\lambda_{a}^{k}\right)^{\prime}=-u_{k} u_{a}
$$

for all $k=1, \ldots, r$ and $a=r+1, \ldots, m-1$. Using (19), (21) and (28), we also get at $s_{0}$

$$
\begin{aligned}
\Psi_{j}^{\prime}= & u_{j}^{\prime} \Phi-2 \sum_{a=r+1}^{m-1} \varepsilon_{a} u_{j} u_{a} \xi_{a}+\sum_{k=1}^{r} \sum_{a=r+1}^{m-1} \varepsilon_{a}\left(u_{j} \lambda_{a}^{k}-u_{k} \lambda_{a}^{j}\right) \Gamma_{a}^{k} \\
\Psi_{j}^{\prime \prime}= & u_{j}^{\prime \prime} \Phi+\sum_{a=r+1}^{m-1} \varepsilon_{a}\left(-3 u_{j}^{\prime} u_{a}+\sum_{k=1}^{r} \varepsilon_{k} u_{k}\left(u_{j} \lambda_{a}^{k}-u_{k} \lambda_{a}^{j}\right)\right) \xi_{a} \\
& +\sum_{k=1}^{r} \sum_{a=r+1}^{m-1} \varepsilon_{a}\left(2 u_{j}^{\prime} \lambda_{a}^{k}-u_{k}^{\prime} \lambda_{a}^{j}+2 u_{j} u_{k} u_{a}\right) \Gamma_{a}^{k} .
\end{aligned}
$$

Since $\Phi^{\prime}=-\sum_{a} \varepsilon_{a} u_{a} \xi_{a}+\sum_{k} \sum_{a} \varepsilon_{a} \lambda_{a}^{k} \Gamma_{a}^{k}$, the functions $\eta_{j}(s)$ and $\vartheta_{j}(s)$ are identically zero on $M_{2}$ for all $j=1, \ldots, r$, with the help of (19), (21), (22) and (29). Thus, we have

$$
\ll \Phi^{\prime}, \Phi \gg=\ll \Phi^{\prime}, \Phi^{\prime} \gg=\ll \Phi^{\prime}, \Phi^{\prime \prime} \gg=\ll \Phi^{\prime}, \Psi_{j} \gg=\ll \Phi^{\prime}, \Psi_{j}^{\prime} \gg=\ll \Phi^{\prime}, \Psi_{j}^{\prime \prime} \gg=0
$$

which means that $\Phi^{\prime}$ is orthogonal to all vectors of (24) except the constant vector C, so taking the indefinite scalar product with $\Phi^{\prime}$ to (24) yields that

$$
f\left(1+\sum_{j=1}^{r} u_{j} t_{j}\right)^{5} \ll \Phi^{\prime}, \mathbf{C} \gg=f\left(1+\sum_{j=1}^{r} u_{j} t_{j}\right)^{5} \gamma^{\prime}=0
$$

which is a contradiction.

Therefore, we have

$$
\gamma^{\prime}=0
$$

on $\tilde{I}_{1}$. Using (30), (24) implies that

$$
3\left(\sum_{j=1}^{r} u_{j}^{\prime} t_{j}\right)\left(\sum_{j=1}^{r} \eta_{j} t_{j}\right)=\left(1+\sum_{j=1}^{r} u_{j} t_{j}\right)\left(\sum_{j=1}^{r} \vartheta_{j} t_{j}\right)
$$

by taking the indefinite scalar product with $\Phi^{\prime}$. Thus, we can see that

$$
\eta_{j}=0=\vartheta_{j}
$$

as the coefficients of terms containing $t_{j}$ and $t_{j}^{2}$ for $j=1, \ldots, r$, by virtue of $u_{j}^{\prime} \neq 0$. Equations (23), (30) and (31) indicate that $\Phi^{\prime}$ is orthogonal to all vectors of (24), so the coefficient of $\Phi^{\prime}$ has to be identically zero, which yields that $u_{j}^{\prime}=0$ for $j=1,2, \ldots, r$. It contradicts our assumption. 
Therefore, we conclude that the functions $u_{j}$ are constant for all $j=1, \ldots, r$, that is,

$$
\frac{\partial q}{\partial s}=0
$$

on $\left(\tilde{I}_{1}\right)$.

Step 2. An expression for $f$ on $\tilde{I}_{1}$.

According to Step 1, Equation (24) is simplified as

$$
\begin{aligned}
\left(\Phi^{\prime \prime}\right. & \left.+\sum_{j=1}^{r} \Psi_{j}^{\prime \prime} t_{j}\right)+\left(\sum_{i=1}^{r} \varepsilon_{i} u_{i}^{2}\right)\left(\Phi+\sum_{j=1}^{r} \Psi_{j} t_{j}\right)-\left(1+\sum_{j=1}^{r} u_{j} t_{j}\right)\left(\sum_{i=1}^{r} \varepsilon_{i} u_{i} \Psi_{i}\right) \\
& +f\left(1+\sum_{j=1}^{r} u_{j} t_{j}\right)^{2}\left\{\left(\Phi+\sum_{j=1}^{r} \Psi_{j} t_{j}\right)+\left(1+\sum_{j=1}^{r} u_{j} t_{j}\right) \mathbf{C}\right\}=\mathbf{0} .
\end{aligned}
$$

We repeat taking the indefinite scalar product to $\Phi^{\prime}$ to (32) and then we obtain

$$
\begin{aligned}
\left(\sum_{j=1}^{r} \vartheta_{j} t_{j}\right) & +\left(\sum_{i=1}^{r} \varepsilon_{i} u_{i}^{2}\right)\left(\sum_{j=1}^{r} z_{j} t_{j}\right)-\left(1+\sum_{j=1}^{r} u_{j} t_{j}\right)\left(\sum_{i=1}^{r} \varepsilon_{i} u_{i} z_{i}\right) \\
& +f\left(1+\sum_{j=1}^{r} u_{j} t_{j}\right)^{2}\left\{\left(\sum_{j=1}^{r} z_{j} t_{j}\right)+\left(1+\sum_{j=1}^{r} u_{j} t_{j}\right) \gamma^{\prime}\right\}=0 .
\end{aligned}
$$

If $\left(\sum_{j} z_{j} t_{j}\right)+\left(1+\sum_{j} u_{j} t_{j}\right) \gamma^{\prime}=0$, then $\gamma^{\prime}=0=z_{j}$ and hence $\vartheta_{j}=0$ in (33) for all $j=1, \ldots, r$. In this case, $\Phi^{\prime \prime}$ and $\Psi_{j}^{\prime \prime}$ are given by (27) and

$$
\begin{aligned}
\Psi_{j}^{\prime \prime}= & \sum_{a=r+1}^{m-1} \varepsilon_{a}\left(u_{j} \sum_{k=1}^{r} \varepsilon_{k} u_{k} \lambda_{a}^{k}-\sum_{k=1}^{r} \varepsilon_{k} u_{k}^{2} \lambda_{a}^{j}-u_{j} u_{a}^{\prime}+\left(\lambda_{a}^{j}\right)^{\prime \prime}\right) \xi_{a} \\
& +\sum_{k=1}^{r} \sum_{a=r+1}^{m-1} \varepsilon_{a}\left(u_{j} u_{k} u_{a}-2 u_{k}\left(\lambda_{a}^{j}\right)^{\prime}+u_{j}\left(\lambda_{a}^{k}\right)^{\prime}\right) \Gamma_{a}^{k} \\
& +\sum_{k=1}^{r} \sum_{a, b=r+1}^{m-1} \varepsilon_{a} \varepsilon_{b}\left(2\left\langle e_{j}^{\prime \prime}, e_{a}\right\rangle \lambda_{b}^{k}+\left\langle e_{k}^{\prime \prime}, e_{b}\right\rangle \lambda_{a}^{j}\right) \Gamma_{a b^{\prime}}^{k}
\end{aligned}
$$

respectively, where we have put

$$
\Gamma_{a b}^{k}=e_{a} \wedge e_{1} \wedge \cdots \wedge e_{k-1} \wedge e_{b} \wedge e_{k+1} \wedge \cdots \wedge e_{r} .
$$

Together with (27) and (34), (32) yields that the constant vector $\mathbf{C}$ can be expressed as

$$
\begin{aligned}
\mathbf{C}= & -\tilde{\varepsilon} \Phi+\sum_{a=r+1}^{m-1} \tilde{\varepsilon} \varepsilon_{a} \ll \mathbf{C}, \xi_{a} \gg \xi_{a}+\sum_{k=1}^{r} \sum_{a=r+1}^{m-1} \tilde{\varepsilon} \varepsilon_{k} \varepsilon_{a} \ll \mathbf{C}, \Gamma_{a}^{k} \gg \Gamma_{a}^{k} \\
& +\sum_{k=1}^{r} \sum_{a, b=r+1}^{m-1} \tilde{\varepsilon} \varepsilon_{k} \varepsilon_{a} \varepsilon_{b} \ll \mathbf{C}, \Gamma_{a b}^{k} \gg \Gamma_{a b}^{k}
\end{aligned}
$$

and that the equations containing $\ll \mathbf{C}, \xi_{a} \gg$ and $\ll \mathbf{C}, \Gamma_{a}^{k} \gg$ are given by 


$$
\begin{aligned}
& 0=\left(-u_{a}^{\prime}+\sum_{k=1}^{r} \varepsilon_{k} u_{k} \lambda_{a}^{k}\right)+\sum_{j=1}^{r} t_{j}\left(u_{j} \sum_{k=1}^{r} \varepsilon_{k} u_{k} \lambda_{a}^{k}-\sum_{k=1}^{r} \varepsilon_{k} u_{k}^{2} \lambda_{a}^{j}-u_{j} u_{a}^{\prime}+\left(\lambda_{a}^{j}\right)^{\prime \prime}\right) \\
&+\left(\sum_{i=1}^{r} \varepsilon_{i} u_{i}^{2}\right)\left(\sum_{j=1}^{r} t_{j} \lambda_{a}^{j}\right)-\left(1+\sum_{j=1}^{r} u_{j} t_{j}\right)\left(\sum_{i=1}^{r} \varepsilon_{i} u_{i} \lambda_{a}^{i}\right) \\
&+f\left(1+\sum_{j=1}^{r} u_{j} t_{j}\right)^{2}\left\{\left(\sum_{j=1}^{r} t_{j} \lambda_{a}^{j}\right)+\tilde{\varepsilon}\left(1+\sum_{j=1}^{r} u_{j} t_{j}\right) \ll \mathbf{C}, \xi_{a} \gg\right\}, \\
& 0=\left(u_{k} u_{a}+\left(\lambda_{a}^{k}\right)^{\prime}\right)+\sum_{j=1}^{r} t_{j}\left(u_{j} u_{k} u_{a}-2 u_{k}\left(\lambda_{a}^{j}\right)^{\prime}+u_{j}\left(\lambda_{a}^{k}\right)^{\prime}\right)+\tilde{\varepsilon} \varepsilon_{k} f\left(1+\sum_{j=1}^{r} u_{j} t_{j}\right)^{3} \ll \mathbf{C}, \Gamma_{a}^{k} \gg,
\end{aligned}
$$

respectively. Therefore, $\ll \mathbf{C}, \xi_{a} \gg$ and $\ll \mathbf{C}, \Gamma_{a}^{k} \gg$ are of the form

$$
\tilde{\varepsilon} \ll \mathbf{C}, \xi_{a} \gg=\frac{u_{a}^{\prime}\left(1+\sum_{j} u_{j} t_{j}\right)-\sum_{j} t_{j}\left(\lambda_{a}^{j}\right)^{\prime \prime}-f\left(1+\sum_{j} u_{j} t_{j}\right)^{2}\left(\sum_{j} t_{j} \lambda_{a}^{j}\right)}{f\left(1+\sum_{j} u_{j} t_{j}\right)^{3}}
$$

and

$$
\tilde{\varepsilon} \varepsilon_{k} \ll \mathbf{C}, \Gamma_{a}^{k} \gg=-\frac{A^{a k}+\sum_{j} B_{j}^{a k} t_{j}}{f\left(1+\sum_{j} u_{j} t_{j}\right)^{3}}
$$

where

$$
\begin{aligned}
& A^{a k}=\ll \Phi^{\prime \prime}, \Gamma_{a}^{k} \gg=u_{k} u_{a}+\left(\lambda_{a}^{k}\right)^{\prime} \quad \text { and } \\
& B_{j}^{a k}=\ll \Psi_{j}^{\prime \prime}, \Gamma_{a}^{k} \gg=u_{j} u_{k} u_{a}-2 u_{k}\left(\lambda_{a}^{j}\right)^{\prime}+u_{j}\left(\lambda_{a}^{k}\right)^{\prime} .
\end{aligned}
$$

If $\ll \mathbf{C}, \Gamma_{a}^{k} \gg=0$, then $A^{a k}=0=B_{j}^{a k}$ in (37). By definitions of $A^{a k}$ and $B_{j}^{a k},\left(\lambda_{a}^{j}\right)^{\prime}=0$ and hence $u_{j} u_{a}=0$ for all $j=1, \ldots, r$ and $a=r+1, \ldots, m-1$. Thus, we have $u_{a}=0$ and (36) is simplified as

$$
\tilde{\varepsilon} \ll \mathbf{C}, \xi_{a} \gg=-\frac{\left(\sum_{j} t_{j} \lambda_{a}^{j}\right)}{\left(1+\sum_{j} u_{j} t_{j}\right)}
$$

for $a=r+1, \ldots, m-1$. Equation (38) implies that $\ll \mathbf{C}, \xi_{a} \gg=0$ and $\lambda_{a}^{j}=0$ for $j=1, \ldots, r$ and $a=r+1, \ldots, m-1$. Thus, $\Psi_{j}=u_{j} \Phi$ and hence $G=\Phi$. Also, under these conditions, by computation, we get $\ll \Psi_{j}^{\prime \prime}, \Gamma_{a b}^{k} \gg=0$ which implies that $\mathbf{C}=-\tilde{\varepsilon} \Phi$ by virtue of (27), (34) and (35). Therefore, the Gauss map $G$ is a constant vector, a contradiction. Therefore, we see that $\ll \mathbf{C}, \Gamma_{a}^{k} \gg \neq 0$. Then, it follows from (37) that

$$
f\left(1+\sum_{j=1}^{r} u_{j} t_{j}\right)^{3}=h(s)\left(A^{a k}+\sum_{j=1}^{r} B_{j}^{a k} t_{j}\right)
$$

for some non-vanishing function $h$ of $s$. Putting (39) into (36), we have

$$
\begin{aligned}
& \tilde{\varepsilon} \ll \mathbf{C}, \xi_{a} \gg \\
& =\frac{u_{a}^{\prime}\left(1+\sum_{j} u_{j} t_{j}\right)^{2}-\left(1+\sum_{j} u_{j} t_{j}\right)\left(\sum_{j} t_{j}\left(\lambda_{a}^{j}\right)^{\prime \prime}\right)-h(s)\left(A^{a k}+\sum_{j} B_{j}^{a k} t_{j}\right)\left(\sum_{j} t_{j} \lambda_{a}^{j}\right)}{h(s)\left(1+\sum_{j} u_{j} t_{j}\right)\left(A^{a k}+\sum_{j} B_{j}^{a k} t_{j}\right)}
\end{aligned}
$$

which allows us to have the following equation

$$
\begin{aligned}
g(s) & h(s)\left(1+\sum_{j=1}^{r} u_{j} t_{j}\right)\left(A^{a k}+\sum_{j=1}^{r} B_{j}^{a k} t_{j}\right) \\
= & u_{a}^{\prime}\left(1+\sum_{j=1}^{r} u_{j} t_{j}\right)^{2}-\left(1+\sum_{j=1}^{r} u_{j} t_{j}\right)\left(\sum_{j=1}^{r} t_{j}\left(\lambda_{a}^{j}\right)^{\prime \prime}\right)-h(s)\left(A^{a k}+\sum_{j=1}^{r} B_{j}^{a k} t_{j}\right)\left(\sum_{j=1}^{r} t_{j} \lambda_{a}^{j}\right)
\end{aligned}
$$


for some non-vanishing function $g$ of $s$. Comparing the coefficients of terms containing $t_{j}^{0}, t_{j}^{1}$ and $t_{j}^{2}$ of (40) gives us three equations:

$$
\begin{aligned}
& g(s) h(s) A^{a k}=u_{a}^{\prime} \\
& g(s) h(s) u_{j} A^{a k}+g(s) h(s) B_{j}^{a k}=2 u_{j} u_{a}^{\prime}-\left(\lambda_{a}^{j}\right)^{\prime \prime}-h(s) A^{a k} \lambda_{a,}^{j} \\
& g(s) h(s) u_{j} B_{j}^{a k}=u_{j}^{2} u_{a}^{\prime}-u_{j}\left(\lambda_{a}^{j}\right)^{\prime \prime}-h(s) B_{j}^{a k} \lambda_{a}^{j} .
\end{aligned}
$$

Combining these equations, we get

$$
u_{j} A^{a k}=B_{j}^{a k} .
$$

Therefore,

$$
u_{j}\left(u_{k} u_{a}+\left(\lambda_{a}^{k}\right)^{\prime}\right)=u_{j} u_{k} u_{a}-2 u_{k}\left(\lambda_{a}^{j}\right)^{\prime}+u_{j}\left(\lambda_{a}^{k}\right)^{\prime},
$$

that is, $u_{k}\left(\lambda_{a}^{j}\right)^{\prime}=0$ and hence

$$
\left(\lambda_{a}^{j}\right)^{\prime}=0
$$

for all $j, k=1, \ldots, r$ and $a=r+1, \ldots, m-1$. From (39), we can obtain

$$
f=\frac{h(s) A^{a k}}{\left(1+\sum_{j} u_{j} t_{j}\right)^{2}}=\frac{h(s) u_{k} u_{a}}{\left(1+\sum_{j} u_{j} t_{j}\right)^{2}}
$$

and then we have

$$
\begin{aligned}
\left(\Phi^{\prime \prime}\right. & \left.+\sum_{j=1}^{r} \Psi_{j}^{\prime \prime} t_{j}\right)+\left(\sum_{i=1}^{r} \varepsilon_{i} u_{i}^{2}\right)\left(\Phi+\sum_{j=1}^{r} \Psi_{j} t_{j}\right)-\left(1+\sum_{j=1}^{r} u_{j} t_{j}\right)\left(\sum_{i=1}^{r} \varepsilon_{i} u_{i} \Psi_{i}\right) \\
& +h(s) u_{k} u_{a}\left\{\left(\Phi+\sum_{j=1}^{r} \Psi_{j} t_{j}\right)+\left(1+\sum_{j=1}^{r} u_{j} t_{j}\right) \mathbf{C}\right\}=\mathbf{0}
\end{aligned}
$$

from (32). By regarding (43) as the polynomial in $t$ of degree 1 , we get

$$
\begin{gathered}
\Phi^{\prime \prime}+\left(\sum_{i=1}^{r} \varepsilon_{i} u_{i}^{2}\right) \Phi-\left(\sum_{i=1}^{r} \varepsilon_{i} u_{i} \Psi_{i}\right)+h(s) u_{k} u_{a} \Phi+h(s) u_{k} u_{a} \mathbf{C}=\mathbf{0}, \\
\Psi_{j}^{\prime \prime}+\left(\sum_{i=1}^{r} \varepsilon_{i} u_{i}^{2}\right) \Psi_{j}-u_{j}\left(\sum_{i=1}^{r} \varepsilon_{i} u_{i} \Psi_{i}\right)+h(s) u_{k} u_{a} \Psi_{j}+h(s) u_{k} u_{a} u_{j} \mathbf{C}=\mathbf{0}
\end{gathered}
$$

which produce that

$$
\Psi_{j}^{\prime \prime}=u_{j} \Phi^{\prime \prime}-\left(\sum_{i=1}^{r} \varepsilon_{i} u_{i}^{2}\right)\left(\Psi_{j}-u_{j} \Phi\right)-h(s) u_{k} u_{a}\left(\Psi_{j}-u_{j} \Phi\right)
$$

for all $j$. With the help of (17), (27), (34) and (41), the equation above provides that

$$
h(s) u_{k} u_{a}=0
$$

which means that $f=0$ due to (42), a contradiction.

Therefore, we conclude that

$$
\left(\sum_{j=1}^{r} z_{j} t_{j}\right)+\left(1+\sum_{j=1}^{r} u_{j} t_{j}\right) \gamma^{\prime} \neq 0
$$

on $\tilde{I}_{1}$ and hence the function $f$ is given by 


$$
f=-\frac{\left(\sum_{j} \vartheta_{j} t_{j}\right)+\left(\sum_{i} \varepsilon_{i} u_{i}^{2}\right)\left(\sum_{j} z_{j} t_{j}\right)-\left(1+\sum_{j} u_{j} t_{j}\right)\left(\sum_{i} \varepsilon_{i} u_{i} z_{i}\right)}{\left(1+\sum_{j} u_{j} t_{j}\right)^{2}\left(\left(\sum_{j} z_{j} t_{j}\right)+\left(1+\sum_{j} u_{j} t_{j}\right) \gamma^{\prime}\right)}
$$

from (33).

Step 3. We find the another equation for $f$ on $\tilde{I}_{1}$.

First, we suppose that $\ll \mathbf{C}, \Gamma_{a}^{k} \gg=\ll \mathbf{C}, \Gamma_{a b}^{k} \gg=0$ of (35) for all $k=1, \ldots, r$ and $a, b=$ $r+1, \ldots, m-1$. Then, we have

$$
\mathbf{C}=-\tilde{\varepsilon} \Phi+\sum_{a=r+1}^{m-1} \tilde{\varepsilon}_{a} \ll \mathbf{C}, \xi_{a} \gg \xi_{a}
$$

and

$$
\ll \Phi^{\prime \prime}, \Gamma_{a}^{k} \gg=\ll \Phi^{\prime \prime}, \Gamma_{a b}^{k} \gg=\ll \Psi_{j}^{\prime \prime}, \Gamma_{a}^{k} \gg=\ll \Psi_{j}^{\prime \prime}, \Gamma_{a b}^{k} \gg=0
$$

for $k=1, \ldots, r$ and $a, b=r+1, \ldots, m-1$. In this case, $\Phi^{\prime \prime}$ and $\Psi_{j}^{\prime \prime}$ are given by

$$
\begin{aligned}
\Phi^{\prime \prime} & =\sum_{a=r+1}^{m-1} \varepsilon_{a}\left(-u_{a}^{\prime}+\sum_{k=1}^{r} \varepsilon_{k} u_{k} \lambda_{a}^{k}\right) \xi_{a}+\sum_{k=1}^{r} \sum_{a=r+1}^{m-1} \varepsilon_{a}\left(u_{k} u_{a}+\left(\lambda_{a}^{k}\right)^{\prime}\right) \Gamma_{a}^{k} \\
& -2 \sum_{k=1}^{r} \sum_{a, b=r+1}^{m-1} \varepsilon_{a} \varepsilon_{b} u_{a} \lambda_{b}^{k} \Gamma_{a b}^{k} \\
\Psi_{j}^{\prime \prime} & =\sum_{a=r+1}^{m-1} \varepsilon_{a}\left(\left\langle e_{j}^{\prime \prime \prime}, e_{a}\right\rangle+2 u_{j} \sum_{k=1}^{r} \varepsilon_{k} u_{k} \lambda_{a}^{k}-\sum_{k=1}^{r} \varepsilon_{k} u_{k}^{2} \lambda_{a}^{j}\right) \xi_{a} \\
& +\sum_{k=1}^{r} \sum_{a=r+1}^{m-1} \varepsilon_{a}\left(2\left\langle e_{j}^{\prime \prime}, \alpha^{\prime}\right\rangle \lambda_{a}^{k}-2 u_{k}\left\langle e_{j}^{\prime \prime}, e_{a}\right\rangle+u_{j}\left\langle e_{k}^{\prime \prime}, e_{a}\right\rangle-\left\langle e_{k}^{\prime \prime}, \alpha^{\prime}\right\rangle \lambda_{a}^{j}\right) \Gamma_{a}^{k} \\
& +\sum_{k=1}^{r} \sum_{a, b=r+1}^{m-1} \varepsilon_{a} \varepsilon_{b}\left(2\left\langle e_{j}^{\prime \prime}, e_{a}\right\rangle \lambda_{b}^{k}+\left\langle e_{k}^{\prime \prime}, e_{b}\right\rangle \lambda_{a}^{j}\right) \Gamma_{a b}^{k} .
\end{aligned}
$$

With the help of (46), the first three equations of (45) provide

$$
\left(\lambda_{a}^{k}\right)^{\prime}=-u_{k} u_{a}, \quad u_{a} \lambda_{b}^{k}=u_{b} \lambda_{a}^{k}, \quad \text { and } \quad u_{a}=0
$$

for all $k=1, \ldots, r$ and $a, b=r+1, \ldots, m-1$.

Taking the indefinite scalar product with $\xi_{a}$ to (32) gives us the equation containing $\ll \mathbf{C}, \xi_{a} \gg$ in the following

$$
\begin{aligned}
& \ll \Phi^{\prime \prime}, \xi_{a} \gg+\sum_{j=1}^{r} t_{j} \ll \Psi_{j}^{\prime \prime}, \xi_{a} \gg+\left(\sum_{i=1}^{r} \varepsilon_{i} u_{i}^{2}\right) \sum_{j=1}^{r} t_{j} \ll \Psi_{j}, \xi_{a} \gg \\
& -\left(1+\sum_{j=1}^{r} u_{j} t_{j}\right)\left(\sum_{i=1}^{r} \varepsilon_{i} u_{i} \ll \Psi_{j}, \xi_{a} \gg\right) \\
& +f\left(1+\sum_{j=1}^{r} u_{j} t_{j}\right)^{2}\left\{\left(\sum_{j=1}^{r} t_{j} \ll \Psi_{j}, \xi_{a} \gg\right)+\left(1+\sum_{j=1}^{r} u_{j} t_{j}\right) \ll \mathbf{C}, \xi_{a} \gg\right\}=0 .
\end{aligned}
$$

Using (46) and (47), Equation (48) is rewritten as

$$
f\left(1+\sum_{j=1}^{r} u_{j} t_{j}\right)^{2}\left\{\left(\sum_{j=1}^{r} \lambda_{a}^{j} t_{j}\right)+\tilde{\varepsilon}\left(1+\sum_{j=1}^{r} u_{j} t_{j}\right) \ll \mathbf{C}, \xi_{a} \gg\right\}=0
$$

which gives 


$$
\left(\sum_{j=1}^{r} \lambda_{a}^{j} t_{j}\right)+\tilde{\varepsilon}\left(1+\sum_{j=1}^{r} u_{j} t_{j}\right) \ll \mathbf{C}, \xi_{a} \gg=0
$$

and hence

$$
\lambda_{a}^{j}=0 \text { and } \ll \mathbf{C}, \xi_{a} \gg=0
$$

for all $j=1, \ldots, r$ and $a=r+1, \ldots, m-1$. Thus, $G=\Phi=-\tilde{\varepsilon} \mathbf{C}$, a contradiction. Therefore, we conclude that $\ll \mathbf{C}, \Gamma_{a}^{k} \gg \neq 0$ or $\ll \mathbf{C}, \Gamma_{a b}^{k} \gg \neq 0$ for some $k, a$ and $b$. Now we assume that $\ll \mathbf{C}, \Gamma_{a}^{k} \gg \neq 0$. Then, taking the indefinite scalar product with $\Gamma_{a}^{k}$ to (32), we obtain

$$
\ll \Phi^{\prime \prime}, \Gamma_{a}^{k} \gg+\sum_{j=1}^{r} t_{j} \ll \Psi_{j}^{\prime \prime}, \Gamma_{a}^{k} \gg+f\left(1+\sum_{j=1}^{r} u_{j} t_{j}\right)^{3} \ll \mathbf{C}, \Gamma_{a}^{k} \gg=0,
$$

or, equivalently,

$$
f=-\frac{A^{a k}+\sum_{j} B_{j}^{a k} t_{j}}{\left(1+\sum_{j} u_{j} t_{j}\right)^{3} \ll \mathbf{C}, \Gamma_{a}^{k} \gg} .
$$

Comparing two Equations (44) and (49) regarding the function $f$, we get

$$
\begin{aligned}
& \left.\left(A^{a k}+\sum_{j=1}^{r} B_{j}^{a k} t_{j}\right)\left\{\left(\sum_{j=1}^{r} z_{j} t_{j}\right)+\left(1+\sum_{j=1}^{r} u_{j} t_{j}\right) \gamma^{\prime}\right)\right\} \\
& =\left(1+\sum_{j=1}^{r} u_{j} t_{j}\right) \ll \mathbf{C}, \Gamma_{a}^{k} \gg\left\{\left(\sum_{j=1}^{r} \vartheta_{j} t_{j}\right)+\left(\sum_{i=1}^{r} \varepsilon_{i} u_{i}^{2}\right)\left(\sum_{j=1}^{r} z_{j} t_{j}\right)-\left(1+\sum_{j=1}^{r} u_{j} t_{j}\right)\left(\sum_{i=1}^{r} \varepsilon_{i} u_{i} z_{i}\right)\right\}
\end{aligned}
$$

which provides that

$$
\begin{gathered}
A^{a k} \gamma^{\prime}=-\ll \mathbf{C}, \Gamma_{a}^{k} \gg\left(\sum_{i=1}^{r} \varepsilon_{i} u_{i} z_{i}\right), \\
A^{a k} z_{j}+\gamma^{\prime} u_{j} A^{a k}+\gamma^{\prime} B_{j}^{a k}=\ll \mathbf{C}, \Gamma_{a}^{k} \gg\left(\vartheta_{j}+z_{j}\left(\sum_{i=1}^{r} \varepsilon_{i} u_{i}^{2}\right)-2 u_{j}\left(\sum_{i=1}^{r} \varepsilon_{i} u_{i} z_{i}\right)\right), \\
z_{j} B_{j}^{a k}+\gamma^{\prime} u_{j} B_{j}^{a k}=u_{j} \ll \mathbf{C}, \Gamma_{a}^{k} \gg\left(\vartheta_{j}+z_{j}\left(\sum_{i=1}^{r} \varepsilon_{i} u_{i}^{2}\right)-u_{j}\left(\sum_{i=1}^{r} \varepsilon_{i} u_{i} z_{i}\right)\right)
\end{gathered}
$$

as the coefficients of terms containing $t_{j}^{0}, t_{j}$ and $t_{j}^{2}$ for $j=1, \ldots, r$. Combining these three equations above, we have

$$
z_{j}\left(B_{j}^{a k}-u_{j} A^{a k}\right)=0
$$

for all $j, k=1, \ldots, r$ and $a, b=r+1, \ldots, m-1$. If $z_{j}=0$ for some $j$, then we can see that $\gamma^{\prime}=0$ by applying the same arguments used to show that $\gamma^{\prime}=0$ on $\tilde{I}_{1}$. But, it contradicts $\left(\sum_{j} z_{j} t_{j}\right)+(1+$ $\left.\sum_{j} u_{j} t_{j}\right) \gamma^{\prime} \neq 0$. Therefore, we have

$$
B_{j}^{a k}=u_{j} A^{a k}
$$

and hence the function $f$ becomes

$$
f=-\frac{A^{a k}}{\ll \mathbf{C}, \Gamma_{a}^{k} \gg\left(1+\sum_{j} u_{j} t_{j}\right)^{2}}
$$

for all $k=1, \ldots, r$ and $a=r+1, \ldots, m-1$.

Step 4. We compare the equations for $f$ obtained in Steps 2 and 3.

Putting (50) into (32) and then considering the coefficients of terms containing $t_{j}^{0}$ and $t_{j}$ in the equation obtained in such a way, we get 


$$
\Psi_{j}^{\prime \prime}=u_{j} \Phi^{\prime \prime}+\left(\frac{A^{a k}}{\ll \mathbf{C}, \Gamma_{a}^{k} \gg}-\sum_{i=1}^{r} \varepsilon_{i} u_{i}^{2}\right)\left(\sum_{a=r+1}^{m-1} \varepsilon_{a} \lambda_{a}^{j} \xi_{a}\right)
$$

for $j=1, \ldots, r$. With the help of (46), Equation (51) gives that $\ll \Psi_{j}^{\prime \prime}, \Gamma_{a b}^{k} \gg=u_{j} \ll \Phi^{\prime \prime}, \Gamma_{a b}^{k} \gg$, so we see that $\ll \Psi_{j}^{\prime \prime}, \Gamma_{a b}^{k} \gg=0$ if and only if $\ll \Phi^{\prime \prime}, \Gamma_{a b}^{k} \gg=0$.

If $\ll \Phi^{\prime \prime}, \Gamma_{a b}^{k} \gg=0$, then $u_{a} \lambda_{b}^{k}=u_{b} \lambda_{a}^{k}$ because of (46). In this case,

$$
\begin{aligned}
u_{a} z_{j} & =u_{a} \sum_{b=r+1}^{m-1} \varepsilon_{b} u_{b} \lambda_{b}^{j}=\sum_{b=r+1}^{m-1} \varepsilon_{b} u_{b}\left(u_{a} \lambda_{b}^{j}\right)=\sum_{b=r+1}^{m-1} \varepsilon_{b} u_{b}\left(u_{b} \lambda_{a}^{j}\right) \\
& =\lambda_{a}^{j} \sum_{b=r+1}^{m-1} \varepsilon_{b} u_{b}^{2}=-\lambda_{a}^{j} \phi=0
\end{aligned}
$$

which means that $u_{a}=0$ or $z_{j}=0$ for $j=1, \ldots, r$ and $a=r+1, \ldots, m-1$. The case of $u_{a}=0$ also guarantees $z_{j}=0$, a contradiction. Therefore, we see that $\ll \Psi_{j}^{\prime \prime}, \Gamma_{a b}^{k} \gg \neq 0$ and $\ll \Phi^{\prime \prime}, \Gamma_{a b}^{k} \gg \neq 0$ for all $k=1, \ldots, r$ and $a, b=r+1, \ldots, m-1$. By computation, equation $B_{j}^{a k}=u_{j} A^{a k}$ gives us

$$
2 u_{k}\left(\lambda_{a}^{j}\right)^{\prime}=\left(\sum_{b=r+1}^{m-1} \varepsilon_{b} u_{b} \lambda_{b}^{k}\right) \lambda_{a}^{j}
$$

and equation $\ll \Psi_{j}^{\prime \prime}, \Gamma_{a b}^{k} \gg=u_{j} \ll \Phi^{\prime \prime}, \Gamma_{a b}^{k} \gg$ provides

$$
2\left(\lambda_{a}^{j}\right)^{\prime} \lambda_{b}^{k}-u_{k} u_{b} \lambda_{a}^{j}+\left(\lambda_{b}^{k}\right)^{\prime} \lambda_{a}^{j}-2\left(\lambda_{b}^{j}\right)^{\prime} \lambda_{a}^{k}+u_{k} u_{a} \lambda_{b}^{j}-\left(\lambda_{a}^{k}\right)^{\prime} \lambda_{b}^{j}=0
$$

for all $j, k, a$ and $b$. In particular, by replacing $k$ with $j$ in (53), we have

$$
\left(\lambda_{a}^{j}\right)^{\prime} \lambda_{b}^{j}-\left(\lambda_{b}^{j}\right)^{\prime} \lambda_{a}^{j}+u_{j}\left(u_{a} \lambda_{b}^{j}-u_{b} \lambda_{a}^{j}\right)=0
$$

which implies that

$$
\ll \Phi^{\prime \prime}, \Gamma_{a b}^{k} \gg=u_{a} \lambda_{b}^{j}-u_{b} \lambda_{a}^{j}=0
$$

by virtue of (52). This is a contradiction.

According to Steps 1, 2, 3 and 4, we can conclude that the subset $\tilde{I}_{1}$ is empty, that is, we may assume that $1+\gamma \neq 0$ on $M$.

By Lemma 3, we can see that the function $f$ of (13) is a rational function in $t$ with functions in $s$ as coefficients of the form

$$
f(t)=-\frac{P(t)}{q^{3}\left(1+\sum_{j} u_{j} t_{j}\right)+q^{\frac{7}{2}} \gamma(s)} .
$$

If we substitute (54) into (11) and multiply $\left(1+\sum_{j} u_{j} t_{j}\right)$ by the equation obtained in such a way, then we have 


$$
\begin{aligned}
\left(1+\sum_{j=1}^{r} u_{j} t_{j}\right)\left\{-\frac{3}{2} q\left(\frac{\partial q}{\partial s}\right)\left(\Phi^{\prime}+\sum_{j=1}^{r} \Psi_{j}^{\prime} t_{j}\right)+q^{2}\left(\Phi^{\prime \prime}+\sum_{j=1}^{r} \Psi_{j}^{\prime \prime} t_{j}\right)-\frac{1}{2} q^{2} \sum_{i=1}^{r} \varepsilon_{i} \frac{\partial q}{\partial t_{i}} \Psi_{i}\right\} \\
-\left(\Phi+\sum_{j=1}^{r} \Psi_{j} t_{j}\right)\left\{-\frac{3}{2} q\left(\frac{\partial q}{\partial s}\right)\left(\sum_{j=1}^{r} p_{j} t_{j}\right)+q^{2}\left(\tilde{\varepsilon} \phi+\sum_{j=1}^{r} \tilde{\varepsilon} \varphi_{j} t_{j}\right)-\frac{1}{2} q^{2} \sum_{i=1}^{r} \varepsilon_{i} \frac{\partial q}{\partial t_{i}} u_{i}\right\} \\
=-q^{\frac{1}{2}} \gamma(s)\left\{\left(\frac{\partial q}{\partial s}\right)^{2}\left(\Phi+\sum_{j=1}^{r} \Psi_{j} t_{j}\right)-\frac{3}{2} q\left(\frac{\partial q}{\partial s}\right)\left(\Phi^{\prime}+\sum_{j=1}^{r} \Psi_{j}^{\prime} t_{j}\right)-\frac{1}{2} q\left(\frac{\partial^{2} q}{\partial s^{2}}\right)\left(\Phi+\sum_{j=1}^{r} \Psi_{j} t_{j}\right)\right. \\
+q^{2}\left(\Phi^{\prime \prime}+\sum_{j=1}^{r} \Psi_{j}^{\prime \prime} t_{j}\right)+\frac{1}{2} q \sum_{i=1}^{r} \varepsilon_{i}\left(\frac{\partial q}{\partial t_{i}}\right)^{2}\left(\Phi+\sum_{j=1}^{r} \Psi_{j} t_{j}\right)-\frac{1}{2} q^{2} \sum_{i=1}^{r} \varepsilon_{i} \frac{\partial q}{\partial t_{i}} \Psi_{i} \\
\left.-\frac{1}{2} q^{2} \sum_{i=1}^{r} \varepsilon_{i}\left(\frac{\partial^{2} q}{\partial t_{i}^{2}}\right)\left(\Phi+\sum_{j=1}^{r} \Psi_{j} t_{j}\right)\right\}+q^{\frac{1}{2}} \mathbf{C} P(t) .
\end{aligned}
$$

Next, we will show that the function $q$ is independent of the parameter $s$ and it is a form of perfect square expression in $t$ of degree 2.

We suppose that $q^{\frac{1}{2}}$ is not a polynomial in $t$. Then we have

$$
\begin{aligned}
& \left(1+\sum_{j=1}^{r} u_{j} t_{j}\right)\left\{-\frac{3}{2} q\left(\frac{\partial q}{\partial s}\right)\left(\Phi^{\prime}+\sum_{j=1}^{r} \Psi_{j}^{\prime} t_{j}\right)+q^{2}\left(\Phi^{\prime \prime}+\sum_{j=1}^{r} \Psi_{j}^{\prime \prime} t_{j}\right)-\frac{1}{2} q^{2} \sum_{i=1}^{r} \varepsilon_{i} \frac{\partial q}{\partial t_{i}} \Psi_{i}\right\} \\
& \quad-\left(\Phi+\sum_{j=1}^{r} \Psi_{j} t_{j}\right)\left\{-\frac{3}{2} q\left(\frac{\partial q}{\partial s}\right)\left(\sum_{j=1}^{r} p_{j} t_{j}\right)+q^{2}\left(\tilde{\varepsilon} \phi+\sum_{j=1}^{r} \tilde{\varepsilon} \varphi_{j} t_{j}\right)-\frac{1}{2} q^{2} \sum_{i=1}^{r} \varepsilon_{i} \frac{\partial q}{\partial t_{i}} u_{i}\right\}=\mathbf{0} .
\end{aligned}
$$

By following the same argument to prove Lemma 3.4 in [19], (56) implies that

$$
\frac{\partial q}{\partial s}=0
$$

Then, we deduce from (55) the following

$$
\begin{aligned}
& \gamma(s)\left\{q\left(\Phi^{\prime \prime}+\sum_{j=1}^{r} \Psi_{j}^{\prime \prime} t_{j}\right)+\frac{1}{2} \sum_{i=1}^{r} \varepsilon_{i}\left(\left(\frac{\partial q}{\partial t_{i}}\right)^{2}-\frac{\partial^{2} q}{\partial t_{i}^{2}}\right)\left(\Phi+\sum_{j=1}^{r} \Psi_{j} t_{j}\right)-\frac{1}{2} q \sum_{i=1}^{r} \varepsilon_{i} \frac{\partial q}{\partial t_{i}} \Psi_{i}\right\} \\
& =\mathbf{C}\left\{q\left(\tilde{\varepsilon} \phi+\sum_{j=1}^{r} \tilde{\varepsilon} \varphi_{j} t_{j}\right)+\frac{1}{2} \sum_{i=1}^{r} \varepsilon_{i}\left(\left(\frac{\partial q}{\partial t_{i}}\right)^{2}-\frac{\partial^{2} q}{\partial t_{i}^{2}}\right)\left(1+\sum_{j=1}^{r} u_{j} t_{j}\right)-\frac{1}{2} q \sum_{i=1}^{r} \varepsilon_{i} \frac{\partial q}{\partial t_{i}} u_{i}\right\},
\end{aligned}
$$

or,

$$
\frac{1}{2} \sum_{i=1}^{r} \varepsilon_{i}\left(\frac{\partial q}{\partial t_{i}}\right)^{2}\left\{\gamma(s) \Phi-\mathbf{C}+\sum_{j=1}^{r}\left(\gamma(s) \Psi_{j}-u_{j} \mathbf{C}\right) t_{j}\right\}=q(t) \Gamma(t),
$$

where $\Gamma(t)$ is a polynomial in $t$ such that $\operatorname{deg} \Gamma=1$ with vector functions of $s$ as coefficients. Considering the degrees of (57), we see that

$$
\sum_{i=1}^{r} \varepsilon_{i}\left(\frac{\partial q}{\partial t_{i}}\right)^{2}=c q(t)
$$

for some constant $c$.

Suppose that there exist $j_{1}, \ldots, j_{l} \in\{1, \ldots, r\}$ such that $\left(\frac{\partial q}{\partial t_{j_{k}}}\right)^{2}$ are not a multiple of $q(t)$ for $k=1, \ldots, l$. By (58), we get

$$
\sum_{k=1}^{l} \varepsilon_{j_{k}}\left(\frac{\partial q}{\partial t_{j_{k}}}\right)^{2}=c_{1} q(t)
$$


for some constant $c_{1}$. By hypothesis, we can put

$$
\left(\frac{\partial q}{\partial t_{j_{k}}}\right)^{2}=c_{j_{k}} q(t)+r_{j_{k}}(t)
$$

for some constants $c_{j_{k}}$ and polynomials $r_{j_{k}}(t)$ in $t$ with $\operatorname{deg} r_{j_{k}}(t) \leq 1$ for $k=1, \ldots, l$. Then, $\sum_{k=1}^{l} \varepsilon_{j_{k}} r_{j_{k}}(t)$ has to be a multiple of $q(t)$ because of (59), a contradiction. Thus, we have

$$
\left(\frac{\partial q}{\partial t_{i}}\right)^{2}=4 u_{i}^{2} q(t)
$$

which yields

$$
w_{i j}=u_{i} u_{j}
$$

by comparing the both sides of (60) for all $i, j=1, \ldots, r$. It contradicts that $q^{\frac{1}{2}}$ is not a polynomial. Therefore, we have

$$
q=\left(1+\sum_{j=1}^{r} u_{j} t_{j}\right)^{2}
$$

for all $s$ and $t$.

Since $1+\gamma(s) \neq 0$ on $M$, the rational function $f$ defined by (54) becomes

$$
f=\frac{P(t)}{\left(1+\sum_{j} u_{j} t_{j}\right)^{7}(1+\gamma(s))} .
$$

Lemma 4. Let $M$ be an $(r+1)$-dimensional non-cylindrical and non-planar ruled submanifold parameterized by (3) in $\mathbb{L}^{m}$. Let $e_{1}, e_{2}, \ldots, e_{r}$ be the orthonormal generators of the rulings along the base curve $\alpha$ such that $e_{j}^{\prime}$ are non-null for all $j=1,2, \ldots$, r. If $M$ has pointwise 1-type Gauss map of the second kind, then we have

$$
e_{j}^{\prime}=u_{j} \alpha^{\prime}
$$

for all $j=1,2, \ldots, r$.

Proof. If $M$ is Lorentzian, it is obvious. We now suppose that $M$ is space-like. In this case,

$$
\varepsilon_{j}=1 \quad \text { and } \quad q=\left(1+\sum_{j=1}^{r} u_{j} t_{j}\right)^{2}
$$

for all $j=1, \ldots, r$. Therefore, (55) can be rewritten as

$$
3\left(\sum_{j=1}^{r} u_{j}^{\prime} t_{j}\right)\left(\sum_{j=1}^{r}\left(p_{j}+\gamma(s) u_{j}^{\prime}\right) t_{j}\right)\left(\Phi+\sum_{j=1}^{r} \Psi_{j} t_{j}\right)=\left(1+\sum_{j=1}^{r} u_{j} t_{j}\right) \Gamma_{1}(t),
$$

where we have put

$$
\begin{aligned}
\Gamma_{1}(t)= & 3\left(\sum u_{j}^{\prime} t_{j}\right)\left(\Phi^{\prime}+\sum \Psi_{j}^{\prime} t_{j}\right)-\left(1+\sum u_{j} t_{j}\right)\left(\Phi^{\prime \prime}+\sum \Psi_{j}^{\prime \prime} t_{j}\right) \\
& +\left(1+\sum u_{j} t_{j}\right)^{2}\left(\sum u_{i} \Psi_{i}\right)+\left(\phi+\sum \varphi_{j} t_{j}\right)\left(\Phi+\sum \Psi_{j} t_{j}\right) \\
& -\left(1+\sum u_{j} t_{j}\right)\left(\sum u_{i}^{2}\right)\left(\Phi+\sum \Psi_{j} t_{j}\right)+3 \gamma\left(\sum u_{j}^{\prime} t_{j}\right)\left(\Phi^{\prime}+\sum \Psi_{j}^{\prime} t_{j}\right) \\
& +\gamma\left(\sum u_{j}^{\prime \prime} t_{j}\right)\left(\Phi+\sum \Psi_{j} t_{j}\right)-\gamma\left(1+\sum u_{j} t_{j}\right)\left(\Phi^{\prime \prime}+\sum \Psi_{j}^{\prime \prime} t_{j}\right) \\
& -\gamma\left(1+\sum u_{j} t_{j}\right)\left(\sum u_{i}^{2}\right)\left(\Phi+\sum \Psi_{j} t_{j}\right)+\gamma\left(1+\sum u_{j} t_{j}\right)^{2}\left(\sum u_{i} \Psi_{i}\right) \\
& +3\left(\sum u_{j}^{\prime} t_{j}\right)^{2} \mathbf{C}-3\left(\sum u_{j}^{\prime} t_{j}\right)\left(\sum p_{j} t_{j}\right) \mathbf{C}-\left(1+\sum u_{j} t_{j}\right)\left(\sum u_{j}^{\prime \prime} t_{j}\right) \mathbf{C} \\
& +\left(1+\sum u_{j} t_{j}\right)\left(\phi+\sum \varphi_{j} t_{j}\right) \mathbf{C} .
\end{aligned}
$$


According to (63), we may have put

$$
\Gamma_{1}(t)=\Lambda_{0}(s)+\sum_{j=1}^{r} \Lambda_{j}(s) t_{j}+\sum_{j, k=1}^{r} \Lambda_{j k}(s) t_{j} t_{k}
$$

where $\Lambda_{0}, \Lambda_{j}$ and $\Lambda_{j k}$ are vector functions of $s$ for $j, k=1, \ldots, r$. Then, by considering the degrees of polynomials (62) and (64) in $t$, we can see that

$$
\Lambda_{0}(s)=\mathbf{0} \quad \text { and } \quad \Lambda_{j}(s)=\mathbf{0}
$$

which implies that

$$
3 u_{j}^{\prime}\left(p_{j}+\gamma(s) u_{j}^{\prime}\right) \Phi=\Lambda_{j j} \quad \text { and } \quad 3 u_{j}^{\prime}\left(p_{j}+\gamma(s) u_{j}^{\prime}\right) \Psi_{j}=u_{j} \Lambda_{j j}
$$

for all $j=1, \ldots, r$. From the above two equations, we have

$$
u_{j}^{\prime}\left(p_{j}+\gamma(s) u_{j}^{\prime}\right)\left(\Psi_{j}-u_{j} \Phi\right)=\mathbf{0}
$$

If $\Psi_{j}-u_{j} \Phi \equiv \mathbf{0}$, then $\lambda_{a}^{j}=0$ in (17), that is, $e_{j}^{\prime}=u_{j} \alpha^{\prime}$ for all $j=1, \ldots, r$ and $a=r+1, \ldots, m-1$. Now, we consider $J_{1}=\left\{s \in I \mid \Psi_{j}-u_{j} \Phi \neq \mathbf{0}\right\}$ and suppose that $J_{1} \neq \varnothing$. Then, on $J_{1}$,

$$
u_{j}^{\prime}\left(p_{j}+\gamma(s) u_{j}^{\prime}\right)=0
$$

and hence

$$
\Lambda_{j j}=\mathbf{0} .
$$

With the help of (63) and (64), the relations $\Lambda_{0}=\Lambda_{j}=\Lambda_{j j}=0$ provide us with the following results

$$
\begin{gathered}
\phi \mathbf{C}=(1+\gamma)\left\{\Phi^{\prime \prime}-\sum u_{i} \Psi_{i}+\left(\sum u_{i}^{2}\right) \Phi\right\}-\phi \Phi, \\
3 u_{j}^{\prime}\left(u_{j}^{\prime}-p_{j}\right) \mathbf{C}=-3(1+\gamma) u_{j}^{\prime}\left(\Psi_{j}^{\prime}-u_{j} \Phi^{\prime}\right)+\left(\Psi_{j}-u_{j} \Phi\right)\left(u_{j} \phi-\gamma u_{j}^{\prime \prime}-\varphi_{j}\right)
\end{gathered}
$$

for $j=1, \ldots, r$. Considering the orthogonality of vectors of the right sides in (67) and (68), we can see that the $p_{j}=\ll \Psi_{j}^{\prime}, \Phi \gg$ must be zero. Therefore, it follows from (66) that

$$
\left(u_{j}^{\prime}\right)^{2} \gamma=0
$$

Case 1. If $\gamma \neq 0$ on some open interval $J_{2}\left(\subset J_{1}\right)$, then $u_{j}^{\prime}=0$ on $J_{2}$. Then, (55) is simplified as

$$
\begin{aligned}
& \left(1+\sum u_{j} t_{j}\right)\left(\Phi^{\prime \prime}+\sum \Psi_{j}^{\prime \prime} t_{j}\right)-\left(1+\sum u_{j} t_{j}\right)^{2}\left(\sum u_{i} \Psi_{i}\right) \\
& -\left(\phi+\sum \varphi_{j} t_{j}\right)\left(\Phi+\sum \Psi_{j} t_{j}\right)+\left(1+\sum u_{j} t_{j}\right)\left(\sum u_{i}^{2}\right)\left(\Phi+\sum \Psi_{j} t_{j}\right) \\
= & -\gamma\left(1+\sum u_{j} t_{j}\right)\left(\Phi^{\prime \prime}+\sum \Psi_{j}^{\prime \prime} t_{j}\right)-\gamma\left(1+\sum u_{j} t_{j}\right)\left(\sum u_{i}^{2}\right)\left(\Phi+\sum \Psi_{j} t_{j}\right) \\
& +\gamma\left(1+\sum u_{j} t_{j}\right)^{2}\left(\sum u_{i} \Psi_{i}\right)+\left(1+\sum u_{j} t_{j}\right)\left(\phi+\sum \varphi_{j} t_{j}\right) \mathbf{C},
\end{aligned}
$$

or,

$$
\left(\phi+\sum_{j=1}^{r} \varphi_{j} t_{j}\right)\left(\Phi+\sum_{j=1}^{r} \Psi_{j} t_{j}\right)=\left(1+\sum_{j=1}^{r} u_{j} t_{j}\right) \Gamma_{2}(t),
$$

where $\Gamma_{2}$ is a polynomial in $t$ with vector functions of $s$ as coefficients.

If $\Gamma_{2}(t)=\left\{a(s)+\sum_{j} b_{j}(s) t_{j}\right\} Y(s)$ for some functions $a, b_{j}$ of $s$ and a vector $\mathrm{Y}$ of $s$, then Equation (71) gives us

$$
\phi \Phi=a \mathrm{Y}
$$




$$
\begin{aligned}
\phi \Psi_{j}+\varphi_{j} \Phi & =a u_{j} \mathrm{Y}+b_{j} \mathrm{Y}, \\
\varphi_{j} \Psi_{j} & =u_{j} b_{j} \mathrm{Y}
\end{aligned}
$$

as the coefficients of terms containing $t_{j}^{0}, t_{j}^{1}$ and $t_{j}^{2}$ for $j=1, \ldots, r$. Putting (72) into (73) and substituting the equation obtained in such a way into (74), we get

$$
\left(\varphi_{j}-u_{j} \phi\right)\left(\Psi_{j}-u_{j} \Phi\right)=\mathbf{0}
$$

which implies that $\varphi_{j}-u_{j} \phi=0$ on $J_{2}$.

If $\Gamma_{2}$ is of the form $\Gamma_{2}=Y_{0}(s)+\sum_{j} Y_{j}(s) t_{j}$ for some vectors $Y_{0}$ and $Y_{j}$ along $s$, we also have the only possible case of $\left(\phi+\sum \varphi_{j} t_{j}\right)=\phi\left(1+\sum u_{j} t_{j}\right)$.

Then, the condition of $\varphi_{j}=u_{j} \phi$ renders (70) simple as follows

$$
\begin{gathered}
(1+\gamma)\left\{\Phi^{\prime \prime}+\sum \Psi_{j}^{\prime \prime} t_{j}-\left(\sum u_{i} \Psi_{i}\right)\left(1+\sum u_{j} t_{j}\right)+\left(\sum u_{i}^{2}\right)\left(\Phi+\sum \Psi_{j} t_{j}\right)\right\} \\
=\phi\left\{\Phi+\sum \Psi_{j} t_{j}+\left(1+\sum u_{j} t_{j}\right) \mathbf{C}\right\} .
\end{gathered}
$$

Here, we may assume that $\phi \neq 0$. If not, that is, $\phi=0=\varphi_{j}$, it follows from (75) that

$$
\Phi^{\prime \prime}+\sum \Psi_{j}^{\prime \prime} t_{j}=\left(\sum u_{i} \Psi_{i}\right)\left(1+\sum u_{j} t_{j}\right)-\left(\sum u_{i}^{2}\right)\left(\Phi+\sum \Psi_{j} t_{j}\right)
$$

because of $1+\gamma \neq 0$. By computations, $u_{j}^{\prime}=0$ and (76) implies that $\Delta G=0$. According to Theorem 3.4 in [9], we can see that it is part of an $(r+1)$-plane in $\mathbb{L}^{m}$.

Considering the constant terms with respect to $t$ and the coefficients of terms containing $t_{j}$ of (75), we have

$$
\begin{gathered}
(1+\gamma)\left\{\Phi^{\prime \prime}-\sum u_{i} \Psi_{i}+\left(\sum u_{i}^{2}\right) \Phi\right\}=\phi(\Phi+\mathbf{C}), \\
(1+\gamma)\left\{\Psi_{j}^{\prime \prime}-u_{j}\left(\sum u_{i} \Psi_{i}\right)+\left(\sum u_{i}^{2}\right) \Psi_{j}\right\}=\phi\left(\Psi_{j}+u_{j} \mathbf{C}\right) .
\end{gathered}
$$

Differentiating (17) with respect to $s$ gives

$$
\Psi_{j}^{\prime \prime}=u_{j} \Phi^{\prime \prime}+\sum_{a=r+1}^{m-1} \varepsilon_{a}\left(\lambda_{a}^{j}\right)^{\prime \prime} \xi_{a}+2 \sum_{a=r+1}^{m-1} \varepsilon_{a}\left(\lambda_{a}^{j}\right)^{\prime} \xi_{a}^{\prime}+\sum_{a=r+1}^{m-1} \varepsilon_{a} \lambda_{a}^{j} \xi_{a}^{\prime \prime} .
$$

Here,

$$
\begin{aligned}
\xi_{a}^{\prime}= & e_{a}^{\prime} \wedge e_{1} \wedge \cdots \wedge e_{r}+\sum_{i=1}^{r} e_{a} \wedge e_{1} \wedge \cdots \wedge e_{i}^{\prime} \wedge \cdots \wedge e_{r} \\
\xi_{a}^{\prime \prime}= & e_{a}^{\prime \prime} \wedge e_{1} \wedge \cdots \wedge e_{r}+2 \sum_{i=1}^{r} e_{a}^{\prime} \wedge e_{1} \wedge \cdots \wedge e_{i}^{\prime} \wedge \cdots \wedge e_{r} \\
& +\sum_{k, l=1}^{r} e_{a} \wedge e_{1} \wedge \cdots \wedge e_{k}^{\prime} \wedge \cdots \wedge e_{l}^{\prime} \wedge \cdots \wedge e_{r}+\sum_{i=1}^{r} e_{a} \wedge e_{1} \wedge \cdots \wedge e_{i}^{\prime \prime} \wedge \cdots \wedge e_{r} .
\end{aligned}
$$

In (80), we can see that the vector $e_{a} \wedge e_{1} \wedge \cdots \wedge e_{k}^{\prime} \wedge \cdots \wedge e_{l}^{\prime} \wedge \cdots \wedge e_{r}$ of $\xi_{a}^{\prime \prime}$ is orthogonal to $\xi_{a}$ and other vectors in (80) except the vectors having the same form. Note that

$$
\begin{aligned}
\ll e_{a} \wedge e_{1} & \wedge \cdots \wedge e_{k}^{\prime} \wedge \cdots \wedge e_{l}^{\prime} \wedge \cdots \wedge e_{r}, e_{b} \wedge e_{1} \wedge \cdots \wedge e_{k}^{\prime} \wedge \cdots \wedge e_{l}^{\prime} \wedge \cdots \wedge e_{r} \gg \\
& =\left|\begin{array}{ccc}
0 & \lambda_{a}^{k} & \lambda_{a}^{l} \\
\lambda_{b}^{k} & u_{k}^{2} & u_{k} u_{l} \\
\lambda_{b}^{l} & u_{k} u_{l} & u_{l}^{2}
\end{array}\right|=u_{l}^{2} \lambda_{a}^{k} \lambda_{b}^{k}+u_{k} u_{l}\left(\lambda_{a}^{k} \lambda_{b}^{l}+\lambda_{a}^{l} \lambda_{b}^{k}\right)-u_{k}^{2} \lambda_{a}^{l} \lambda_{b}^{l}
\end{aligned}
$$

for $k, l=1, \ldots, r$ and $a, b=r+1, \ldots, m-1$. 
We multiply $u_{j}$ by (77) and then compare (78) and the equation obtained in such a way. Then, we can obtain

$$
\begin{aligned}
\phi \sum_{a=r+1}^{m-1} \varepsilon_{a} \lambda_{a}^{j} \xi_{a}=(1+\gamma(s))\{ & \sum_{a=r+1}^{m-1} \varepsilon_{a}\left(\lambda_{a}^{j}\right)^{\prime \prime} \xi_{a}+2 \sum_{a=r+1}^{m-1} \varepsilon_{a}\left(\lambda_{a}^{j}\right)^{\prime} \xi_{a}^{\prime} \\
& \left.+\sum_{a=r+1}^{m-1} \varepsilon_{a} \lambda_{a}^{j} \xi_{a}^{\prime \prime}+\left(\sum_{i=1}^{r} u_{i}^{2}\right) \sum_{a=r+1}^{m-1} \varepsilon_{a} \lambda_{a}^{j} \xi_{a}\right\}
\end{aligned}
$$

with the help of (17) and (79). By taking the wedge product with $e_{k}$ to (82) for some $k,(80)$ and (82) induce the following

$$
\begin{aligned}
\mathbf{0}= & 2 \sum_{a=r+1}^{m-1} \varepsilon_{a}\left(\lambda_{a}^{j}\right)^{\prime} e_{a} \wedge e_{1} \wedge \cdots \wedge e_{k}^{\prime} \wedge \cdots \wedge e_{r} \wedge e_{k} \\
& +2 \sum_{a=r+1}^{m-1} \varepsilon_{a} \lambda_{a}^{j} e_{a}^{\prime} \wedge e_{1} \wedge \cdots \wedge e_{k}^{\prime} \wedge \cdots \wedge e_{r} \wedge e_{k} \\
& +\sum_{a=r+1}^{m-1} \varepsilon_{a} \lambda_{a}^{j} e_{a} \wedge e_{1} \wedge \cdots \wedge e_{k}^{\prime \prime} \wedge \cdots \wedge e_{r} \wedge e_{k} \\
& +\sum_{a=r+1}^{m-1} \varepsilon_{a} \lambda_{a}^{j}\left(\sum_{i \neq k}^{r} e_{a} \wedge e_{1} \wedge \cdots \wedge e_{k}^{\prime} \wedge \cdots \wedge e_{i}^{\prime} \wedge \cdots \wedge e_{r} \wedge e_{k}\right) .
\end{aligned}
$$

Again, taking the wedge product with $e_{i}$ to (83) for $i \neq k$, we get

$$
\varepsilon_{a} \lambda_{a}^{j} e_{a} \wedge e_{1} \wedge \cdots \wedge e_{k}^{\prime} \wedge \cdots \wedge e_{i}^{\prime} \wedge \cdots \wedge e_{r} \wedge e_{k} \wedge e_{i}=\mathbf{0}
$$

for all $j=1, \ldots, r$ and $a=r+1, \ldots, m-1$. If $\lambda_{a}^{j} \neq 0$ for some $j$ and $a$, then putting (16) into (84) implies that

$$
u_{k} \lambda_{b}^{i}-u_{i} \lambda_{b}^{k}=0
$$

for $b=r+1, \ldots, m-1$. Here, we note that $\left(e_{k}^{\prime}\right)^{\perp} \wedge\left(e_{i}^{\prime}\right)^{\perp}=\mathbf{0}$ because of $w_{i j}=u_{i} u_{k}$. Using (85), we can see that the value of (81) becomes zero, which means that the coefficients of $\xi_{a}^{\prime \prime}$ in (82) must be identically zero by the orthogonality of vectors. Therefore, we have $\lambda_{a}^{j}=0$ and hence $e_{j}^{\prime}=u_{j} \alpha^{\prime}$ on $J_{2}$, for all $j$ and $a$.

Case 2. We consider $A=\{s \in I \mid \gamma(s)=0\}$ and suppose that $\operatorname{Int}(A) \neq \varnothing$. Then, on $\operatorname{Int}(A)$,

$$
0=\ll \Phi, \mathbf{C} \gg=\ll \Phi^{\prime}, \mathbf{C} \gg
$$

and Equation (68) is simplified as

$$
3\left(u_{j}^{\prime}\right)^{2} \mathbf{C}=-3 u_{j}^{\prime}\left(\Psi_{j}^{\prime}-u_{j} \Phi^{\prime}\right)+\left(\Psi_{j}-u_{j} \Phi\right)\left(u_{j} \phi-\varphi_{j}\right) .
$$

With the help of (86), taking the indefinite scalar product with $\Phi^{\prime}$ to (87) gives

$$
0=-3 u_{j}^{\prime}\left(\ll \Psi_{j}^{\prime}, \Phi^{\prime} \gg-u_{j} \ll \Phi^{\prime}, \Phi^{\prime} \gg\right)+\ll \Psi_{j}, \Phi^{\prime} \gg\left(u_{j} \phi-\varphi_{j}\right)
$$

which yields

$$
-2 u_{j}^{\prime}\left(u_{j} \phi-\varphi_{j}\right)=0
$$

because of $\ll \Psi_{j^{\prime}}^{\prime} \Phi^{\prime} \gg=-\varphi_{j}, \ll \Phi^{\prime}, \Phi^{\prime} \gg=-\phi$ and $\ll \Psi_{j}, \Phi^{\prime} \gg=u_{j}^{\prime}$. If $u_{j}^{\prime} \neq 0$, then $\varphi_{j}=u_{j} \phi$, which implies that $e_{j}^{\prime}=u_{j} \alpha^{\prime}$ by applying the same arguments used in Case 1 . If $u_{j}^{\prime}=0$ on $\operatorname{Int}(A)$, then, by continuity, $u_{j}^{\prime}=0$ on $A$ and hence $e_{j}^{\prime}=u_{j} \alpha^{\prime}$ on $A$ for the same reasons as Case 1 .

According to Cases 1 and 2, we can conclude that 


$$
e_{j}^{\prime}=u_{j} \alpha^{\prime}
$$

on $J_{1}$ and hence on $M$. This proof is complete.

From Lemma 4, the Gauss map $G$ is given by

$$
G=\Phi
$$

and thus $\Delta G=f(G+\mathbf{C})$ yields

$$
\frac{1}{2 q^{2}} \frac{\partial q}{\partial s} \Phi^{\prime}-\frac{1}{q} \Phi^{\prime \prime}=f(\Phi+\mathbf{C})
$$

Taking the indefinite scalar product to (88) with $\Phi$, we obtain

$$
-\frac{1}{q} \phi(s)=f(\tilde{\varepsilon}+\gamma(s)) .
$$

Suppose that $\phi(s) \equiv 0$ on $I$. Then we have two cases concerning $\Phi^{\prime}$.

If $\Phi^{\prime} \equiv \mathbf{0}$ on $I$, then the Gauss map $G$ is a constant vector field and hence $M$ is an open part of an $(r+1)$-plane in $\mathbb{L}^{m}$.

Now, we suppose that $\Phi^{\prime}$ is null on some interval $U$. Then the normal part of $\alpha^{\prime \prime}$ of (18) has to be null on $U$ as well. Therefore, we have $\varepsilon_{j}=1$ for $j=1, \ldots, r$. By (89), we get

$$
f(1+\gamma(s))=0
$$

Since $1+\gamma(s) \neq 0$, we see that $f=0$ on $U$. Then, Equation (88) is rewritten as

$$
\left(\sum_{j=1}^{r} \varepsilon u_{j}^{\prime} t_{j}\right) \Phi^{\prime}-\left(1+\sum_{j=1}^{r} \varepsilon u_{j} t_{j}\right) \Phi^{\prime \prime}=\mathbf{0}
$$

which yields

$$
\Phi^{\prime \prime}=\mathbf{0} \quad \text { and } \quad u_{j}^{\prime}=0
$$

for all $j=1, \ldots, r$. By the definition of $\Phi$ and Lemma 4 , we have

$$
\mathbf{0}=\Phi^{\prime \prime}=\alpha^{\prime \prime \prime} \wedge e_{1} \wedge \cdots \wedge e_{r}+\sum_{k=1}^{r} \alpha^{\prime \prime} \wedge e_{1} \wedge \cdots \wedge e_{k-1} \wedge e_{k}^{\prime} \wedge e_{k+1} \wedge \cdots \wedge e_{r} .
$$

Taking the wedge product with $e_{k}$ to (90) for some $k$, we obtain

$$
\begin{aligned}
\mathbf{0} & =\alpha^{\prime \prime} \wedge e_{1} \wedge \cdots \wedge e_{k-1} \wedge e_{k}^{\prime} \wedge e_{k+1} \wedge \cdots \wedge e_{r} \wedge e_{k} \\
& =\alpha^{\prime \prime} \wedge e_{1} \wedge \cdots \wedge e_{k-1} \wedge u_{k} \alpha^{\prime} \wedge e_{k+1} \wedge \cdots \wedge e_{r} \wedge e_{k} .
\end{aligned}
$$

Without loss of generality, we may assume that $u_{k}$ is a non-zero constant. So we have

$$
\alpha^{\prime \prime} \wedge e_{1} \wedge \cdots \wedge e_{k-1} \wedge \alpha^{\prime} \wedge e_{k+1} \wedge \cdots \wedge e_{r} \wedge e_{k}=\mathbf{0}
$$

which means that $\alpha^{\prime \prime}$ is tangent to $M$, a contradiction.

Therefore, we conclude that if $\phi(s) \equiv 0$ on $I$, then $\Phi^{\prime} \equiv \mathbf{0}$ and $M$ is part of an $(r+1)$-plane in $\mathbb{L}^{m}$.

We now suppose that the open subset $J=\{s \in I \mid \phi(s) \neq 0\}$ is not empty. Then, we may put

$$
f=-\frac{\phi(s)}{q(\tilde{\varepsilon}+\gamma(s))} .
$$


Using $q=\left(1+\sum_{i} \varepsilon u_{i} t_{i}\right)^{2}$ and putting (91) into (88), we have

$$
\left(\sum_{j=1}^{r} u_{j}^{\prime} t_{j}\right) \Phi^{\prime}-\left(1+\sum_{j=1}^{r} u_{j} t_{j}\right) \Phi^{\prime \prime}=-\frac{\phi}{(\tilde{\varepsilon}+\gamma(s))}\left(1+\sum_{j=1}^{r} u_{j} t_{j}\right)(\Phi+\mathbf{C}) .
$$

In (92), considering the constant terms with respect to $t$ and the coefficients of terms containing $t_{j}$, we see

$$
\Phi^{\prime \prime}=\frac{\phi}{(\tilde{\varepsilon}+\gamma(s))}(\Phi+\mathbf{C})
$$

and hence

$$
u_{j}^{\prime} \Phi^{\prime}=\mathbf{0}
$$

for all $j=1,2, \ldots, r$. Since $\Phi^{\prime}(s) \neq \mathbf{0}, u_{j}$ are constant for all $j=1,2, \ldots, r$ and $s \in J$. Together with Lemma 4, we have

Lemma 5. Let $M$ be an $(r+1)$-dimensional non-cylindrical and non-planar ruled submanifold parameterized by (3) in $\mathbb{L}^{m}$ with pointwise 1-type Gauss map of the second kind. Let $e_{1}, e_{2}, \ldots, e_{r}$ be the orthonormal generators of the rulings along the base curve $\alpha$. If $e_{j}^{\prime}$ are non-null for all $j=1,2, \ldots, r$, then the functions

$$
u_{j}(s)=\left\langle\alpha^{\prime}, e_{j}^{\prime}\right\rangle \text { and } w_{i j}(s)=\left\langle e_{i}^{\prime}, e_{j}^{\prime}\right\rangle
$$

are constant functions on the open interval $J=\{s \in I \mid \phi(s) \neq 0\}$ for all $i, j=1,2, \ldots, r$, where $\phi(s)=\ll$ $\Phi(s), \Phi^{\prime \prime}(s) \gg$.

Furthermore, for the case that $e_{j}^{\prime}$ are non-null for all $j$, we have

Lemma 6. Let $M$ be an $(r+1)$-dimensional non-cylindrical and non-planar ruled submanifold parameterized by (3) in $\mathbb{L}^{m}$. We suppose that $e_{j}^{\prime}$ are non-null for all $j=1,2, \ldots, r$. If $M$ has pointwise 1-type Gauss map of the second kind, we can choose an orthonormal frame $\left\{e_{a}\right\}_{a=r+1}^{m-1}$ of the normal space $\left(T_{\alpha(s)} M\right)^{\perp}$ of $M$ along $\alpha$ satisfying

$$
e_{a}^{\prime} \wedge \alpha^{\prime}(s)=0
$$

for all $a=r+1, \ldots, m-1$.

Proof. It is sufficient to see Lemma 3.5 in [20].

Proposition 1. Let $M$ be an $(r+1)$-dimensional non-cylindrical and non-planar ruled submanifold parameterized by (3) in $\mathbb{L}^{m}$. Let $e_{1}, e_{2}, \ldots, e_{r}$ be the orthonormal generators of the rulings along the base curve $\alpha$ such that $e_{j}^{\prime}$ are non-null for all $j=1,2, \ldots, r$. If $M$ has pointwise 1-type Gauss map of the second kind, then the parametrization of $M$ is given by

$$
x\left(s, t_{1}, \ldots, t_{r}\right)=t_{1} \beta(s)+\sum_{i=2}^{r} t_{i} \boldsymbol{a}_{i}+\boldsymbol{D},
$$

where $\beta(s)$ is part of a circle or a hyperbola in $\mathbb{L}^{m}$ with $\boldsymbol{a}_{2}, \boldsymbol{a}_{3}, \ldots, \boldsymbol{a}_{r}$ orthonormal constant vectors satisfying $\left\langle\beta^{\prime}(s), \boldsymbol{a}_{i}\right\rangle=\left\langle\beta(s), \boldsymbol{a}_{i}\right\rangle=0, \boldsymbol{D}$ a constant vector and $t_{i} \in I_{i}$ for some open intervals $I_{i}$ and $i=2, \ldots, r$.

Proof. Suppose $\phi(s) \equiv 0$ on the whole domain $I$ of $\alpha$. In this case, we showed that $M$ is part of an $(r+1)$ - plane in $\mathbb{L}^{m}$. Clearly, a plane can be parameterized as (94) for some suitable constant vectors $\mathbf{a}_{2}, \ldots, \mathbf{a}_{r}$.

Now, we suppose that $M$ is not part of an $(r+1)$-plane, that is, $J=\{s \in I \mid \phi(s) \neq 0\}$ is not empty. Note that 


$$
\ll \Phi^{\prime}, \Phi^{\prime \prime} \gg=-\frac{\phi^{\prime}}{2}, \quad \ll \Phi^{\prime}, \Phi \gg=0 \quad \text { and } \quad \phi=-\tilde{\varepsilon} \sum_{a=r+1}^{m-1} \varepsilon_{a} u_{a}^{2} .
$$

Then, Equation (93) implies

$$
-\frac{\phi^{\prime}}{2}=\frac{\phi}{\tilde{\varepsilon}+\gamma} \ll \Phi^{\prime}, \mathbf{C} \gg=\frac{\phi}{\tilde{\varepsilon}+\gamma} \gamma^{\prime},
$$

or, equivalently,

$$
-\frac{1}{2} \frac{\phi^{\prime}}{\phi}=\frac{(\tilde{\varepsilon}+\gamma)^{\prime}}{(\tilde{\varepsilon}+\gamma)}
$$

on J. Equation (96) yields

$$
\frac{1}{\sqrt{|\phi|}}=\tilde{\lambda}|\tilde{\varepsilon}+\gamma|
$$

for some positive constant $\tilde{\lambda}$. Therefore, from (93) we get

$$
\Phi^{\prime \prime}=\lambda \sqrt{|\phi|^{3}}(\Phi+\mathbf{C})
$$

for some non-zero constant $\lambda$.

Meanwhile, according to Lemma 6, we can put

$$
\alpha^{\prime \prime}=-\sum_{i=1}^{r} \varepsilon_{i} u_{i} e_{i}-\sum_{a=r+1}^{m-1} \varepsilon_{a} u_{a} e_{a} \quad \text { and } \quad e_{a}^{\prime}=\varepsilon u_{a} \alpha^{\prime}
$$

for all $a=r+1, \ldots, m-1$. Since $\frac{1}{\lambda \sqrt{|\phi|^{3}}} \Phi^{\prime \prime}-\Phi$ is constant, by straightforward computation, we have

$$
\begin{aligned}
\mathbf{0}= & \sum_{a=r+1}^{m-1} \varepsilon_{a}\left\{\left(\frac{u_{a}^{\prime}}{\lambda \sqrt{|\phi|^{3}}}\right)^{\prime} u_{a}-u_{a}\left(\frac{\varepsilon \mu}{\lambda \sqrt{|\phi|^{3}}}+1\right)\right\} \xi_{a} \\
& +\sum_{j=1}^{r} \sum_{a=r+1}^{m-1} \varepsilon_{a} u_{j}\left\{\left(\frac{1}{\lambda \sqrt{|\phi|^{3}}}\right)^{\prime} u_{a}+\left(\frac{2}{\lambda \sqrt{|\phi|^{3}}}\right) u_{a}^{\prime}\right\} e_{a} \wedge e_{1} \wedge \cdots \wedge e_{j-1} \wedge \alpha^{\prime} \wedge e_{j+1} \wedge \cdots \wedge e_{r},
\end{aligned}
$$

where $\mu=\left\langle\alpha^{\prime \prime}, \alpha^{\prime \prime}\right\rangle$. By the orthogonality of the vectors $\alpha^{\prime}, e_{j}$ and $e_{a}$ for all $j=1, \ldots, r$ and $a=$ $r+1, \ldots, m-1,(98)$ yields

$$
\left(\frac{1}{\lambda \sqrt{|\phi|^{3}}}\right)^{\prime} u_{a}+\left(\frac{2}{\lambda \sqrt{|\phi|^{3}}}\right) u_{a}^{\prime}=0
$$

for all $a=r+1, \ldots, m-1$. Since $\phi \neq 0$ on $J$ and $\phi=-\tilde{\varepsilon} \sum_{a} \varepsilon_{a} u_{a}^{2}$, there exists a non-zero function $u_{b}$ for some $b=r+1, \ldots, m-1$. Then, (99) implies

$$
\frac{3}{4} \frac{|\phi|^{\prime}}{|\phi|}=\frac{u_{b}^{\prime}}{u_{b}} .
$$

So we can see that

$$
|\phi|^{\frac{3}{4}}=\lambda_{b} u_{b} \quad \text { or, } \quad u_{b}^{2}=\frac{1}{\lambda_{b}^{2}}|\phi|^{\frac{3}{2}}
$$

for some non-zero real number $\lambda_{b}$. By (95), we have

$$
\phi=c|\phi|^{\frac{3}{2}}
$$


for some negative constant $c$, which means that the function $\phi$ is constant and hence the functions $u_{a}$ are constant for all $a=r+1, \ldots, m-1$ by virtue of (99). By continuity, the interval $J$ is the whole domain $I$ of $\alpha$. Furthermore, (97) implies

$$
\alpha^{\prime \prime \prime}=-\mu \alpha^{\prime}
$$

for the constant $\mu=\sum_{i} \varepsilon_{i} u_{i}^{2}+\sum_{a} \varepsilon_{a} u_{a}^{2}$. By Lemma 2, we can see that the curve $\alpha$ is contained in a 2-dimensional subspace of $\mathbb{L}^{m}$. Equation (100) gives that the curvature is non-zero constant and hence the plane curve $\alpha$ is part of a circle or a hyperbola.

Considering Lemmas 4-6, we may put

$$
\alpha(s)=\frac{\varepsilon}{u_{1}}\left(e_{1}-\mathbf{a}_{1}\right) \quad \text { and } \quad e_{i}(s)=\frac{u_{i}}{u_{1}} e_{1}(s)+\mathbf{b}_{i}
$$

for some constant vectors $\mathbf{a}_{1}$ and $\mathbf{b}_{i}$ for $i=2, \ldots, r$ such that $e_{1}(s), \mathbf{b}_{2}, \mathbf{b}_{3}, \ldots, \mathbf{b}_{r}$ are linearly independent for each $s$. By applying Gram-Schmidt's orthogonalization, we get orthonormal constant vectors $\mathbf{a}_{2}, \ldots, \mathbf{a}_{r}$ from $\mathbf{b}_{2}, \ldots, \mathbf{b}_{r} .\left\langle e_{1}(s), \mathbf{b}_{i}\right\rangle$ are constant and thus $\left\langle e_{1}(s), \mathbf{a}_{i}\right\rangle$ are also constant for all $i=2, \ldots, r$.

We put $v_{i}=\left\langle e_{1}(s), \mathbf{a}_{i}\right\rangle$ for all $i=2, \ldots, r$. Define

$$
\beta_{1}(s)=e_{1}(s)-\sum_{r=2}^{r} \tau_{i} v_{i} \mathbf{a}_{i}
$$

where $\tau_{i}=\left\langle\mathbf{a}_{i}, \mathbf{a}_{i}\right\rangle(= \pm 1)$. Then $\left\langle\beta_{1}(s), \beta_{1}(s)\right\rangle=\varepsilon_{1}-\sum_{i=2}^{r} \tau_{i} v_{i}^{2}$ is a non-zero constant since $e_{1}(s), \mathbf{a}_{2}$, $\ldots, \mathbf{a}_{r}$ are linearly independent. Take $\beta(s)=\frac{\beta_{1}(s)}{\| \beta_{1}(s)||}$, where $\left\|\beta_{1}(s)\right\|=\sqrt{\left|\left\langle\beta_{1}, \beta_{1}\right\rangle\right|}$. After appropriate change of parameters $t_{1}, t_{2}, \ldots, t_{r}$, the parametrization of (3) for $M$ can be reduced to

$$
x\left(s, \overline{t_{1}}, \overline{t_{2}}, \ldots, \overline{t_{r}}\right)=\overline{t_{1}} \beta(s)+\sum_{i=2}^{r} \bar{t}_{i} \mathbf{a}_{i}+\mathbf{D}
$$

for some constant vector $\mathbf{D}$.

If $\alpha$ is a circle, we can see that the trace of position vectors of $\beta(s)$ is a circle on the unit sphere by virtue of the first equation of (101).

Note that if $q<0$, that is, $\alpha$ is time-like, then we can see that $\alpha$ is part of a hyperbola in $\mathbb{L}^{m}$ by applying the same arguments developed in the proof of Proposition 1. Therefore, we can also obtain the parametrization (94) for $M$ such that the curve $\beta$ is part of a hyperbola.

We now consider the case that some of the generators of rulings have null derivatives. Let $M$ be an $(r+1)$-dimensional non-cylindrical ruled submanifold parameterized by (3) in $\mathbb{L}^{m}$. Again, if we use Proposition 3.3 of [19], we may assume that $e_{i}^{\prime} \neq \mathbf{0}$ for all $i=1, \ldots, r$.

Case 3. Suppose that $e_{i}^{\prime}$ are null for all $i=1, \ldots, r$. We then have three possible cases according to the degree of $q$.

Subcase 3.1. Let $\operatorname{deg} q(t)=0$, that is, $e_{i}^{\prime}$ are null with $e_{i}^{\prime}(s) \wedge e_{l}^{\prime}(s)=\mathbf{0}$ for $i, l=1,2, \ldots, r$ and $u_{j}=\left\langle\alpha^{\prime}(s), e_{j}^{\prime}(s)\right\rangle=0$ for $j=1,2, \ldots, r$. Note that $\varepsilon_{i}=1$ for all $i=1,2, \ldots, r$. Then $M$ has the Gauss map of the form

$$
G=\Phi+\sum_{j=1}^{r} t_{j} \Psi_{j}
$$

Therefore, $\Delta G=f(G+\mathbf{C})$ implies

$$
\Phi^{\prime \prime}=-f(\Phi+\mathbf{C}) \quad \text { and } \quad \Psi_{j}^{\prime \prime}=-f \Psi_{j}
$$


for all $j=1,2, \ldots, r$. Equation (102) shows that the function $f$ depends on the parameter $s$ only. From $e_{i}^{\prime}(s) \wedge e_{j}^{\prime}(s)=\mathbf{0}$, we can put

$$
e_{j}^{\prime}=\sigma_{j} e_{1}^{\prime}
$$

where $\sigma_{j}$ are non-vanishing functions of $s$ for all $i, j=1,2, \ldots, r$. Also, $e_{j}^{\prime}(s) \wedge e_{j}^{\prime \prime}(s)=\mathbf{0}$ follows from $e_{i}^{\prime}(s) \wedge e_{j}^{\prime}(s)=\mathbf{0}$ and hence we have

$$
e_{j}^{\prime \prime}=h_{j} e_{j}^{\prime}
$$

for some functions $h_{j}$ of $s$ and $j=1,2, \ldots, r$. By (18) and (104), we see

$$
0=u_{j}^{\prime}=\left\langle\alpha^{\prime \prime}, e_{j}^{\prime}\right\rangle=-\sigma_{j} \sum_{a=r+1}^{m-1} \varepsilon_{a} u_{a} \lambda_{a}^{1}
$$

which implies

$$
\sum_{a=r+1}^{m-1} \varepsilon_{a} u_{a} \lambda_{a}^{1}=0 .
$$

By straightforward computation, equation $\Psi_{j}^{\prime \prime}=-f \Psi_{j}$ of (102) provides that

$$
\sigma_{j} h_{j}=\sigma_{j}^{\prime}+\sigma_{j} h_{1} \quad \text { and } \quad-f=h_{j}^{2}+h_{j}^{\prime}
$$

with the help of (103) and (104).

Now, on the non-empty open interval $I_{0}=\{s \in I \mid f(s) \neq 0\}$, the first equation of (102) implies

$$
f^{\prime} \Phi^{\prime \prime}=f \Phi^{\prime \prime \prime}+f^{2} \Phi^{\prime}
$$

In this case, we recall that

$$
\phi=\ll \Phi, \Phi^{\prime \prime} \gg=-\ll \Phi^{\prime}, \Phi^{\prime} \gg=-\left\langle\alpha^{\prime \prime}, \alpha^{\prime \prime}\right\rangle=-\sum_{a=r+1}^{m-1} \varepsilon_{a} u_{a}^{2}
$$

because of $\ll \Phi, \Phi^{\prime} \gg=0$. From the definition of $\Phi$, we get

$$
\begin{gathered}
\Phi^{\prime}=-\sum_{a=r+1}^{m-1} \varepsilon_{a} u_{a} \xi_{a}+\sum_{k=1}^{r} \sigma_{k} \Omega_{1}^{k} \\
\Phi^{\prime \prime}=\phi \Phi-\sum_{a=r+1}^{m-1} \varepsilon_{a} u_{a}^{\prime} \xi_{a}+\sum_{k=1}^{r} \sigma_{k} h_{k} \Omega_{1}^{k}-2 \sum_{k=1}^{r} \sum_{a=r+1}^{m-1} \varepsilon_{a} u_{a} \sigma_{k} \Omega_{a, 1}^{k}, \\
\Phi^{\prime \prime \prime}=\frac{3}{2} \phi^{\prime} \Phi-\sum_{a=r+1}^{m-1} \varepsilon_{a}\left(u_{a} \phi+u_{a}^{\prime \prime}\right) \xi_{a}+\sum_{k=1}^{r}\left(3 \sigma_{k} \phi-f \sigma_{k}\right) \Omega_{1}^{k} \\
-\sum_{k=1}^{r} \sum_{a=r+1}^{m-1} \varepsilon_{a}\left(3 u_{a}^{\prime} \sigma_{k}+3 u_{a} \sigma_{k} h_{k}\right) \Omega_{a, 1}^{k}
\end{gathered}
$$

with the aid of (18), (103)-(105) and (108), where we have put

$$
\begin{aligned}
& \Omega_{1}^{k}=\alpha^{\prime} \wedge e_{1} \wedge \cdots \wedge e_{k-1} \wedge e_{1}^{\prime} \wedge e_{k+1} \wedge \cdots \wedge e_{r} \\
& \Omega_{a, 1}^{k}=e_{a} \wedge e_{1} \wedge \cdots \wedge e_{k-1} \wedge e_{1}^{\prime} \wedge e_{k+1} \wedge \cdots \wedge e_{r} .
\end{aligned}
$$

Considering (107) and (109)-(111), we have the following:

$$
f^{\prime} \phi=\frac{3}{2} f \phi^{\prime},
$$




$$
\begin{gathered}
f^{\prime} \sigma_{k} h_{k}=3 f \sigma_{k} \phi, \\
2 f^{\prime} u_{a} \sigma_{k}=f\left(3 u_{a}^{\prime} \sigma_{k}+3 u_{a} \sigma_{k} h_{k}\right)
\end{gathered}
$$

as the coefficients of vectors $\Phi, \Omega_{1}^{k}$ and $\Omega_{a, 1}^{k}$, respectively, for all $k=1, \ldots, r$ and $a=r+1, \ldots, m-1$. Note that $\sigma_{k}$ are non-vanishing for all $k=1, \ldots, r$. Multiplying (114) by $\varepsilon_{a} u_{a}$ and adding the equations obtained in such a way together with respect to $a$, we obtain

$$
2 f^{\prime} \sum_{a=r+1}^{m-1} \varepsilon_{a} u_{a}^{2}=3 f \sum_{a=r+1}^{m-1} \varepsilon_{a} u_{a} u_{a}^{\prime}+3 f h_{k} \sum_{a=r+1}^{m-1} \varepsilon_{a} u_{a}^{2}
$$

or,

$$
2 f^{\prime} \phi=\frac{3}{2} f \phi^{\prime}+3 f \phi h_{k}
$$

for all $k=1,2, \ldots, r$. By (112), (115) yields that

$$
h_{k}=\frac{1}{2} \frac{\phi^{\prime}}{\phi}
$$

By putting (112) into (113) and then considering (116), we have

$$
h_{k}^{2}=\phi
$$

Thus, $\mu=\left\langle\alpha^{\prime \prime}, \alpha^{\prime \prime}\right\rangle$ is non-positive because of (108). Since $e_{j}^{\prime}$ are null and $\left\langle\alpha^{\prime \prime}, e_{j}^{\prime}\right\rangle=0$ for $j=$ $1, \ldots, r$, the vector $\alpha^{\prime \prime}$ can not be time-like and thus $\phi=0$. Therefore, in (113), we see that

$$
f^{\prime} \sigma_{k} h_{k}=0
$$

for all $k=1, \ldots, r$. If $f^{\prime} \neq 0$, then $h_{k}=0$ and then $f$ is vanishing because of (106), a contradiction on $I_{0}$. Therefore, $f^{\prime}=0$ on $I_{0}$ and hence $f$ is a non-zero constant function on $M$ by continuity. This means that $G$ is of 1-type in the usual sense. For ruled submanifolds with finite-type Gauss map, see [10].

Subcase 3.2. Let $\operatorname{deg} q(t)=1$. In this case, $\left\langle\alpha^{\prime}(s), e_{i}^{\prime}(s)\right\rangle \neq 0$ for some $i(1 \leq i \leq r)$ and the null vector fields $e_{i}^{\prime}$ satisfy $e_{i}^{\prime} \wedge e_{l}^{\prime}=0$ for $i, l=1,2, \ldots, r$. We note that $\tilde{\varepsilon}=1$ and $\varepsilon_{i}=1$ for all $i=1,2, \ldots, r$. Thus, $\Delta G=f(G+\mathbf{C})$ implies

$$
\begin{array}{r}
\left(\frac{\partial q}{\partial s}\right)^{2}\left(1+\sum_{j=1}^{r} u_{j} t_{j}\right)-\frac{3}{2} q \frac{\partial q}{\partial s}\left(\sum_{j=1}^{r} p_{j} t_{j}\right)-\frac{1}{2} q \frac{\partial^{2} q}{\partial s^{2}}\left(1+\sum_{j=1}^{r} u_{j} t_{j}\right) \\
+q^{2}\left(\phi+\sum_{j=1}^{r} \varphi_{j} t_{j}\right)+\frac{1}{2} q \sum_{i=1}^{r}\left(\frac{\partial q}{\partial t_{i}}\right)^{2}\left(1+\sum_{j=1}^{r} u_{j} t_{j}\right)-\frac{1}{2} q^{2} \sum_{i=1}^{r} \frac{\partial q}{\partial t_{i}} u_{i} \\
+f\left\{q^{3}\left(1+\sum_{j=1}^{r} u_{j} t_{j}\right)+q^{\frac{7}{2}} \gamma(s)\right\}=0 .
\end{array}
$$

Note that $q^{3}\left(1+\sum_{j} u_{j} t_{j}\right)+q^{\frac{7}{2}} \gamma(s) \neq 0$ because of $\operatorname{deg} q=1$. Therefore, using the function $f$ obtained from (117), we repeat the same process to get (55). Then, we have the following equation 


$$
\begin{gathered}
\left(1+\sum_{j=1}^{r} u_{j} t_{j}\right)\left\{-\frac{3}{2} q\left(\frac{\partial q}{\partial s}\right)\left(\Phi^{\prime}+\sum_{j=1}^{r} \Psi_{j}^{\prime} t_{j}\right)+q^{2}\left(\Phi^{\prime \prime}+\sum_{j=1}^{r} \Psi_{j}^{\prime \prime} t_{j}\right)-\frac{1}{2} q^{2} \sum_{i=1}^{r} \frac{\partial q}{\partial t_{i}} \Psi_{i}\right\} \\
-\left(\Phi+\sum_{j=1}^{r} \Psi_{j} t_{j}\right)\left\{-\frac{3}{2} q\left(\frac{\partial q}{\partial s}\right)\left(\sum_{j=1}^{r} p_{j} t_{j}\right)+q^{2}\left(\phi+\sum_{j=1}^{r} \varphi_{j} t_{j}\right)-\frac{1}{2} q^{2} \sum_{i=1}^{r} \frac{\partial q}{\partial t_{i}} u_{i}\right\} \\
=-q^{\frac{1}{2}} \gamma(s)\left\{\left(\frac{\partial q}{\partial s}\right)^{2}\left(\Phi+\sum_{j=1}^{r} \Psi_{j} t_{j}\right)-\frac{3}{2} q\left(\frac{\partial q}{\partial s}\right)\left(\Phi^{\prime}+\sum_{j=1}^{r} \Psi_{j}^{\prime} t_{j}\right)-\frac{1}{2} q\left(\frac{\partial^{2} q}{\partial s^{2}}\right)\left(\Phi+\sum_{j=1}^{r} \Psi_{j} t_{j}\right)\right. \\
\left.+q^{2}\left(\Phi^{\prime \prime}+\sum_{j=1}^{r} \Psi_{j}^{\prime \prime} t_{j}\right)+\frac{1}{2} q \sum_{i=1}^{r}\left(\frac{\partial q}{\partial t_{i}}\right)^{2}\left(\Phi+\sum_{j=1}^{r} \Psi_{j} t_{j}\right)-\frac{1}{2} q^{2} \sum_{i=1}^{r} \frac{\partial q}{\partial t_{i}} \Psi_{i}\right\} \\
+q^{\frac{1}{2}} \mathbf{C} P(t),
\end{gathered}
$$

where we have put

$$
\begin{aligned}
P(t)= & \left(\frac{\partial q}{\partial s}\right)^{2}\left(1+\sum_{j=1}^{r} u_{j} t_{j}\right)-\frac{3}{2} q \frac{\partial q}{\partial s}\left(\sum_{j=1}^{r} p_{j} t_{j}\right)-\frac{1}{2} q \frac{\partial^{2} q}{\partial s^{2}}\left(1+\sum_{j=1}^{r} u_{j} t_{j}\right) \\
& +q^{2}\left(\phi+\sum_{j=1}^{r} \varphi_{j} t_{j}\right)+\frac{1}{2} q \sum_{i=1}^{r}\left(\frac{\partial q}{\partial t_{i}}\right)^{2}\left(1+\sum_{j=1}^{r} u_{j} t_{j}\right)-\frac{1}{2} q^{2} \sum_{i=1}^{r} \frac{\partial q}{\partial t_{i}} u_{i} .
\end{aligned}
$$

Since $\operatorname{deg} q=1$, the left side of (118) has to be vanishing, that is,

$$
\begin{gathered}
\left(1+\sum_{j=1}^{r} u_{j} t_{j}\right)\left\{-\frac{3}{2} q\left(\frac{\partial q}{\partial s}\right)\left(\Phi^{\prime}+\sum_{j=1}^{r} \Psi_{j}^{\prime} t_{j}\right)+q^{2}\left(\Phi^{\prime \prime}+\sum_{j=1}^{r} \Psi_{j}^{\prime \prime} t_{j}\right)-\frac{1}{2} q^{2} \sum_{i=1}^{r} \frac{\partial q}{\partial t_{i}} \Psi_{i}\right\} \\
-\left(\Phi+\sum_{j=1}^{r} \Psi_{j} t_{j}\right)\left\{-\frac{3}{2} q\left(\frac{\partial q}{\partial s}\right)\left(\sum_{j=1}^{r} p_{j} t_{j}\right)+q^{2}\left(\phi+\sum_{j=1}^{r} \varphi_{j} t_{j}\right)-\frac{1}{2} q^{2} \sum_{i=1}^{r} \frac{\partial q}{\partial t_{i}} u_{i}\right\}=\mathbf{0} .
\end{gathered}
$$

Using $q=1+\sum_{i} 2 u_{i} t_{i}$ and $\frac{\partial q}{\partial s}=\sum_{i} 2 u_{i}^{\prime} t_{i}$, Equation (119) can be expressed as

$$
\left(\Phi^{\prime}+\sum_{j=1}^{r} \Psi_{j}^{\prime} t_{j}\right)\left(1+\sum_{i=1}^{r} u_{i} t_{i}\right)-\left(\sum_{i=1}^{r} p_{i} t_{i}\right)\left(\Phi+\sum_{j=1}^{r} \Psi_{j} t_{j}\right)=q W(t)
$$

for some vector $W(t)$. In [29], it was proved that Equation (120) implies

$$
\frac{\partial q}{\partial s}=0
$$

Therefore, (119) is simplified as

$$
\begin{aligned}
& \left(1+\sum_{j=1}^{r} u_{j} t_{j}\right)\left(\Phi^{\prime \prime}+\sum_{j=1}^{r} \Psi_{j}^{\prime \prime} t_{j}-\sum_{i=1}^{r} u_{i} \Psi_{i}\right) \\
& -\left(\Phi+\sum_{j=1}^{r} \Psi_{j} t_{j}\right)\left(\phi+\sum_{j=1}^{r} \varphi_{j} t_{j}-\sum_{i=1}^{r} u_{i}^{2}\right)=\mathbf{0}
\end{aligned}
$$

which furnishes us with three equations as follows:

$$
\Phi^{\prime \prime}-\sum_{i=1}^{r} u_{i} \Psi_{i}-\phi \Phi+\left(\sum_{i=1}^{r} u_{i}^{2}\right) \Phi=\mathbf{0}
$$




$$
\begin{gathered}
\Psi_{j}^{\prime \prime}+u_{j} \Phi^{\prime \prime}-u_{j}\left(\sum_{i=1}^{r} u_{i} \Psi_{i}\right)-\varphi_{j} \Phi-\phi \Psi_{j}+\left(\sum_{i=1}^{r} u_{i}^{2}\right) \Psi_{j}=\mathbf{0}, \\
u_{j} \Psi_{j}^{\prime \prime}-\varphi_{j} \Psi_{j}=\mathbf{0}
\end{gathered}
$$

for all $j=1,2, \ldots, r$. Combining (122)-(124), we get

$$
\left(\Psi_{j}-u_{j} \Phi\right)\left(\varphi_{j}-u_{j} \phi+u_{j} \sum_{i=1}^{r} u_{i}^{2}\right)=\mathbf{0}
$$

for all $j=1,2, \ldots, r$. By the characters of $e_{j}^{\prime}$ and $\alpha^{\prime}$, we see that the functions $\lambda_{a}^{j}(s)$ of (16) are non-vanishing for all $s$ and it is impossible to have $\Psi_{j}=u_{j} \Phi$. Thus, we have

$$
\varphi_{j}-u_{j} \phi+u_{j} \sum_{i=1}^{r} u_{i}^{2} \equiv 0
$$

for all $j=1,2, \ldots, r$.

Meanwhile, we note that $e_{j}^{\prime} \wedge e_{i}^{\prime}=\mathbf{0}$ for all $i, j=1, \ldots, r$. Then, we can put

$$
e_{i}^{\prime}=f_{i} e_{j_{0}}^{\prime}
$$

for some $j_{0}$ with $u_{j_{0}} \neq 0$, where $f_{i}$ are non-vanishing functions for all $i=1, \ldots, r$. From the definition of $u_{i}$, we have

$$
u_{i}=f_{i} u_{j_{0}}
$$

which implies that $f_{i}$ are non-zero constant for all $i=1, \ldots, r$. Indeed, we see that $u_{i} \neq 0$ for all $i=1, \ldots, r$. By (14) and (126), we also obtain

$$
\varphi_{i}=f_{i} \varphi_{j_{0}}
$$

for all $i=1, \ldots, r$. Thus, the following vector and function of (121) are induced as

$$
\Phi^{\prime \prime}+\sum_{j=1}^{r} \Psi_{j}^{\prime \prime} t_{j}-\sum_{i=1}^{r} u_{i} \Psi_{i}=\frac{\varphi_{j_{0}}}{u_{j_{0}}}\left(\Phi+\sum_{j=1}^{r} \Psi_{j} t_{j}\right)
$$

and

$$
\phi+\sum_{j=1}^{r} \varphi_{j} t_{j}-\sum_{i=1}^{r} u_{i}^{2}=\frac{\varphi_{j_{0}}}{u_{j_{0}}}\left(1+\sum_{j=1}^{r} u_{j} t_{j}\right)
$$

by virtue of (122), (124), (125), (127) and (128). Using $\frac{\partial q}{\partial s}=0$ and (119), and substituting (129) and (130) into (118), Equation (118) is rewritten as

$$
\left(\frac{\varphi_{j_{0}}}{u_{j_{0}}} q+2 \sum_{i=1}^{r} u_{i}^{2}\right)\left\{\gamma(s)\left(\Phi+\sum_{j=1}^{r} \Psi_{j} t_{j}\right)-\mathbf{C}\left(1+\sum_{j=1}^{r} u_{j} t_{j}\right)\right\}=\mathbf{0} .
$$

We note that $\frac{\varphi_{j_{0}}}{u_{j_{0}}} q+2 \sum_{i} u_{i}^{2}$ of (131) is non-vanishing for all s. If this not the case, since $q=1+$ $\sum_{i} 2 u_{i} t_{i}$ is a polynomial in $t$ of degree 1 and $\sum_{i} u_{i}^{2}$ is constant with respect to $t, \varphi_{j_{0}}$ has to be vanishing for $s$ and hence $\sum_{i} u_{i}^{2}=0$, a contradiction to $u_{i} \neq 0$ for all $i$. Therefore, we have

$$
\gamma(s)\left(\Phi+\sum_{j=1}^{r} \Psi_{j} t_{j}\right)=\mathbf{C}\left(1+\sum_{j=1}^{r} u_{j} t_{j}\right)
$$

or, equivalently, 


$$
\gamma(s) \Phi=\mathbf{C} \quad \text { and } \quad \gamma(s) \Psi_{j}=u_{j} \mathbf{C}
$$

for all $j=1, \ldots, r$. Differentiating ' $\gamma(s) \Phi=\mathbf{C}^{\prime}$ of (132) with respect to $s$ and taking the indefinite scalar product with $\Phi$ to the equation obtained in such a way, we get

$$
\gamma^{\prime}(s)=0
$$

which implies that $\gamma$ is a non-zero constant function for all $s$. If not, that is, $\gamma=0$, the vector $\mathbf{C}$ is zero, a contradiction. Therefore, (132) yields

$$
\Psi_{j}=u_{j} \Phi \quad \text { and hence } \quad e_{j}^{\prime}=u_{j} \alpha^{\prime}
$$

for all $j=1, \ldots, r$. This is also a contradiction to the characters of $e_{j}^{\prime}$ and $\alpha^{\prime}$.

Consequently, we can conclude that there is no ruled submanifold with $\operatorname{deg} q=1$ which has pointwise 1-type Gauss map of the second kind.

Subcase 3.3. Let $\operatorname{deg} q(t)=2$. In this case, we can easily obtain the same conclusion such as Lemma 4 by referring to the case that $e_{1}^{\prime}, e_{2}^{\prime}, \ldots, e_{r}^{\prime}$ are non-null. But this is impossible according to the characters of the vectors $\alpha^{\prime}$ and $e_{j}^{\prime}$. Therefore, we see that no ruled submanifold in $\mathbb{L}^{m}$ with $\operatorname{deg} q=2$ has pointwise 1-type Gauss map of the second kind.

Case 4. Suppose that $e_{j_{1}}^{\prime}, \ldots, e_{j_{k}}^{\prime}$ are null for $j_{1}<j_{2}<\cdots<j_{k} \in\{1,2, \ldots, r\}$ and $e_{i}^{\prime}$ are non-null for $i \neq j_{l}, l=1, \ldots, k$.

In this case, $\operatorname{deg} q=2$. If we follow a similar argument for the case that $e_{1}^{\prime}, e_{2}^{\prime}, \ldots, e_{r}^{\prime}$ are non-null, for the same reason as in Subcase 3.3, we can conclude that there is no ruled submanifold in $\mathbb{L}^{m}$ with pointwise 1-type Gauss map of the second kind under these assumptions.

Until now, we have considered the necessary conditions for ruled submanifolds to have a pointwise 1-type Gauss map of the second kind. That is, if the ruled submanifold $M$ in $\mathbb{L}^{m}$ parameterized by (3) has a pointwise 1-type Gauss map of the second kind, then according to the characters of $e_{i}^{\prime}, M$ is part of a product manifold of a right cone (or a hyperbolic cone) and a plane, or $M$ has a 1-type Gauss map in the usual sense. Conversely, by straightforward computations, we can see that the Gauss maps of these ruled submanifolds are of pointwise 1-type of the second kind.

Therefore, we have

Theorem 2. Let $M$ be an $(r+1)$-dimensional non-cylindrical ruled submanifold with non-degenerate rulings in the Minkowski m-space $\mathbb{L}^{m}$. Then, M has a pointwise 1-type Gauss map $G$ of the second kind if and only if $M$ is one of the following:

(1) M has a 1-type Gauss map in the usual sense, i.e., the Gauss map $G$ satisfies $\Delta G=\lambda G+\mathbf{C}$ for some non-zero $\lambda \in \mathbb{R}$ and some constant vector $\mathbf{C}$.

(2) $M$ is part of a product manifold of a right cone and a plane of the form $\mathbb{C}_{S} \times \mathbb{R}^{r-1}$ or $\mathbb{C}_{S} \times \mathbb{L}^{r-1}$.

(3) $M$ is part of a product manifold of a hyperbolic cone and a plane $\mathbb{C}_{H} \times \mathbb{R}^{r-1}$.

(4) $M$ is part of an $(r+1)$-plane in $\mathbb{L}^{m}$.

\section{Generalized Null Scrolls in $\mathbb{L}^{m}$}

Let $M$ be an $(r+1)$-dimensional ruled submanifold in $\mathbb{L}^{m}$ with degenerate rulings $E(s, r)$ along a regular curve with a parametrization $\tilde{x}(s, t)$, where $t=\left(t_{1}, t_{2}, \ldots, t_{r}\right)$. Since $E(s, r)$ is degenerate, it can be spanned by a degenerate frame $\left\{B(s)=e_{1}(s), e_{2}(s), \ldots, e_{r}(s)\right\}$ such that

$$
\langle B(s), B(s)\rangle=\left\langle B(s), e_{i}(s)\right\rangle=0,\left\langle e_{i}(s), e_{j}(s)\right\rangle=\delta_{i j}, i, j=2,3, \ldots, r .
$$


Without loss of generality as was shown in Lemma 1, we may assume that

$$
\left\langle e_{i}^{\prime}(s), e_{j}(s)\right\rangle=0, i, j=2,3, \cdots, r .
$$

Since the tangent space of $M$ at $\tilde{x}(s, t)$ is non-degenerate and contains the degenerate ruling $E(s, r)$, there exists a tangent vector field $A$ to $M$ which satisfies

$$
\langle A(s, t), A(s, t)\rangle=0,\langle A(s, t), B(s)\rangle=-1,\left\langle A(s, t), e_{i}(s)\right\rangle=0, i=2,3, \ldots, r
$$

at $\tilde{x}(s, t)$.

Let $\alpha(s)$ be an integral curve of the vector field $A$ on $M$. Then we can define another parametrization $x$ of $M$ as follows:

$$
x\left(s, t_{1}, t_{2}, \ldots, t_{r}\right)=\alpha(s)+\sum_{i=1}^{r} t_{i} e_{i}(s),
$$

where $\alpha^{\prime}(s)=A(s)$. A ruled submanifold defined as above is called a generalized null scroll. We refer to two lemmas for later use.

Lemma 7 ([7]). We may assume that $\left\langle A(s), B^{\prime}(s)\right\rangle=0$ for all $s$.

Lemma 8 ([8]). Let $M$ be a ruled submanifold with degenerate rulings. Then, the following are equivalent.

(1) $M$ is minimal

(2) $B^{\prime}$ is tangent to $M$.

If we put $P=\left\langle x_{s}, x_{s}\right\rangle$ and $Q=-\left\langle x_{s}, x_{t_{1}}\right\rangle$, Lemma 7 implies

$$
\begin{gathered}
P(s, t)=2 \sum_{i=2}^{r} u_{i}(s) t_{i}+\sum_{i, j=1}^{r} w_{i j}(s) t_{i} t_{j}, \\
Q(s, t)=1+\sum_{i=2}^{r} v_{i}(s) t_{i}
\end{gathered}
$$

where $v_{i}(s)=\left\langle B^{\prime}(s), e_{i}(s)\right\rangle, u_{i}(s)=\left\langle A(s), e_{i}^{\prime}(s)\right\rangle$ and $w_{i j}(s)=\left\langle e_{i}^{\prime}(s), e_{j}^{\prime}(s)\right\rangle$ for $i, j=1,2, \ldots, r$.

Note that $P$ and $Q$ are polynomials in $t=\left(t_{1}, t_{2}, \ldots, t_{r}\right)$ with functions in $s$ as coefficients. Then the Laplacian $\Delta$ of $M$ can be expressed as follows:

$$
\begin{gathered}
\Delta=\frac{1}{Q^{2}}\left\{\frac{\partial \bar{P}}{\partial t_{1}} \frac{\partial}{\partial t_{1}}-2 Q \sum_{i=2}^{r} v_{i} \frac{\partial}{\partial t_{i}}+2 Q \frac{\partial^{2}}{\partial s \partial t_{1}}+\bar{P} \frac{\partial^{2}}{\partial t_{1}^{2}}\right. \\
\left.-2 Q \sum_{i=2}^{r} v_{i} t_{1} \frac{\partial^{2}}{\partial t_{1} \partial t_{i}}-Q^{2} \sum_{i=2}^{r} \frac{\partial^{2}}{\partial t_{i}^{2}}\right\},
\end{gathered}
$$

where $\bar{P}=P-t_{1}^{2} \sum_{i=2}^{r} v_{i}^{2}$.

By definition of the indefinite scalar product $\ll, \gg$ on $G(r+1, m)$, we may put

$$
\ll x_{s} \wedge x_{t_{1}} \wedge x_{t_{2}} \wedge \cdots \wedge x_{t_{r}}, x_{s} \wedge x_{t_{1}} \wedge x_{t_{2}} \wedge \cdots \wedge x_{t_{r}} \gg=-Q^{2}
$$

Then the Gauss map $G$ is given by 


$$
\begin{aligned}
G= & \frac{1}{|Q|} x_{s} \wedge x_{t_{1}} \wedge x_{t_{2}} \wedge \cdots \wedge x_{t_{r}} \\
= & \frac{1}{|Q|}\left\{A \wedge B \wedge e_{2} \wedge \cdots \wedge e_{r}+t_{1} B^{\prime} \wedge B \wedge e_{2} \wedge \cdots \wedge e_{r}\right. \\
& \left.+\sum_{i=2}^{r} t_{i} e_{i}^{\prime} \wedge B \wedge e_{2} \wedge \cdots \wedge e_{r}\right\} .
\end{aligned}
$$

In [9], the authors proved the following theorem.

Theorem 3 ([9]). Let $M$ be a generalized null scroll in $\mathbb{L}^{m}$. Then, the following are equivalent.

(1) $M$ is minimal.

(2) M has a harmonic Gauss map.

We now suppose that a generalized null scroll $M$ has a pointwise 1-type Gauss map $\Delta G=f(G+$ C). Without loss of generality, we may assume that $Q>0$. Then by straightforward computation, we get

$$
\begin{aligned}
& \frac{2}{Q^{3}} \sum_{h=r+1}^{m-1}\left\{\left(\sum_{i=1}^{r}\left\langle B^{\prime}, e_{i}^{\prime}\right\rangle t_{i}-\sum_{i=2}^{r} v_{i}^{\prime} t_{i}\right) v_{h}+v_{h}^{\prime} Q\right\} e_{h} \wedge B \wedge e_{2} \wedge \cdots \wedge e_{r} \\
+ & \frac{2}{Q^{2}} \sum_{h=r+1}^{m-1} v_{h}^{2} A \wedge B \wedge e_{2} \wedge \cdots \wedge e_{r} \\
& +\frac{2}{Q^{2}} \sum_{i=2}^{r} \sum_{h=r+1}^{m-1} v_{i} v_{h} e_{h} \wedge B \wedge e_{2} \wedge \cdots \wedge e_{i-1} \wedge A \wedge e_{i+1} \wedge \cdots \wedge e_{r} \\
& +\frac{2}{Q^{2}} \sum_{i=2}^{r} \sum_{h, l=r+1}^{m-1} v_{h} \lambda_{l}^{i} e_{h} \wedge B \wedge e_{2} \wedge \cdots \wedge e_{i-1} \wedge e_{l} \wedge e_{i+1} \wedge \cdots \wedge e_{r} \\
= & f\left[\frac{1}{Q}\left\{\left(1+\sum_{i=2}^{r} t_{i} v_{i}\right) A \wedge B \wedge e_{2} \wedge \cdots \wedge e_{r}+\sum_{h=r+1}^{m-1}\left(t_{1} v_{h}+\sum_{i=2}^{r} \lambda_{h}^{i} t_{i}\right) e_{h} \wedge B \wedge e_{2} \wedge \cdots \wedge e_{r}\right\}+\mathbf{C}\right] \\
= & f A \wedge B \wedge e_{2} \wedge \cdots \wedge e_{r}+\frac{f}{Q} \sum_{h=r+1}^{m-1}\left(t_{1} v_{h}+\sum_{i=2}^{r} \lambda_{h}^{i} t_{i}\right) e_{h} \wedge B \wedge e_{2} \wedge \cdots \wedge e_{r}+f \mathbf{C},
\end{aligned}
$$

where we have put

$$
B^{\prime}=\sum_{i=2}^{m-1} v_{i} e_{i} \quad \text { and } \quad e_{j}^{\prime}=v_{j} A-u_{j} B+\sum_{l=r+1}^{m-1} \lambda_{l}^{j} e_{l}
$$

for $j=2, \ldots, r$ and $l=r+1, \ldots, m-1$.

Now, we note that $Q$ is constant with respect to $t_{1}$. Then, by differentiating (133) with respect to $t_{1}$, we get

$$
\begin{aligned}
& \frac{2}{Q^{3}}\left\langle B^{\prime}, B^{\prime}\right\rangle \sum_{h=r+1}^{m-1} v_{h} e_{h} \wedge B \wedge e_{2} \wedge \cdots \wedge e_{r} \\
= & f_{t_{1}} A \wedge B \wedge e_{2} \wedge \cdots \wedge e_{r}+\frac{f_{t_{1}}}{Q} \sum_{h=r+1}^{m-1}\left(t_{1} v_{h}+\sum_{i=2}^{r} \lambda_{h}^{i} t_{i}\right) e_{h} \wedge B \wedge e_{2} \wedge \cdots \wedge e_{r} \\
& +\frac{f}{Q} \sum_{h=r+1}^{m-1} v_{h} e_{h} \wedge B \wedge e_{2} \wedge \cdots \wedge e_{r}+f_{t_{1}} \mathrm{C} .
\end{aligned}
$$

Case 5. $f_{t_{1}} \equiv 0$ on $M$. 
Equation (135) implies that

$$
f=\frac{2}{Q^{2}}\left\langle B^{\prime}, B^{\prime}\right\rangle=\frac{2 w_{11}}{Q^{2}} .
$$

Putting (136) into (133), we obtain the following polynomial in $t$ of degree 1 with functions of $s$ as coefficients

$$
\begin{aligned}
& \sum_{h=r+1}^{m-1}\left\{\left(\sum_{i=1}^{r}\left\langle B^{\prime}, e_{i}^{\prime}\right\rangle t_{i}-\sum_{j=2}^{r} v_{j}^{\prime} t_{j}\right) v_{h}+v_{h}^{\prime} Q\right\} e_{h} \wedge B \wedge e_{2} \wedge \cdots \wedge e_{r} \\
& +Q \sum_{h=r+1}^{m-1} v_{h}^{2} A \wedge B \wedge e_{2} \wedge \cdots \wedge e_{r} \\
& +Q \sum_{j=2}^{r} \sum_{h=r+1}^{m-1} v_{j} v_{h} e_{h} \wedge B \wedge e_{2} \wedge \cdots \wedge e_{j-1} \wedge A \wedge e_{j+1} \wedge \cdots \wedge e_{r} \\
& +Q \sum_{j=2}^{r} \sum_{h, l=r+1}^{m-1} v_{h} \lambda_{l}^{j} e_{h} \wedge B \wedge e_{2} \wedge \cdots \wedge e_{j-1} \wedge e_{l} \wedge e_{j+1} \wedge \cdots \wedge e_{r} \\
& =w_{11} Q A \wedge B \wedge e_{2} \wedge \cdots \wedge e_{r}+w_{11} \sum_{h=r+1}^{m-1}\left(t_{1} v_{h}+\sum_{j=2}^{r} \lambda_{h}^{j} t_{j}\right) e_{h} \wedge B \wedge e_{2} \wedge \cdots \wedge e_{r} \\
& \quad+w_{11} Q \mathbf{C C} .
\end{aligned}
$$

Comparing the constant terms with respect to $t$ of (137) and using $w_{11}=\sum_{j=2}^{r} v_{j}^{2}+\sum_{l=r+1}^{m-1} v_{l}^{2}$, we have

$$
\begin{aligned}
\bar{\varepsilon} w_{11} \mathbf{C}= & \sum_{h=r+1}^{m-1} v_{h}^{\prime} e_{h} \wedge B \wedge e_{2} \wedge \cdots \wedge e_{r}-\sum_{j=2}^{r} v_{j}^{2} A \wedge B \wedge e_{2} \wedge \cdots \wedge e_{r} \\
& +\sum_{j=2}^{r} \sum_{h=r+1}^{m-1} v_{j} v_{h} e_{h} \wedge B \wedge e_{2} \wedge \cdots \wedge e_{j-1} \wedge A \wedge e_{j+1} \wedge \cdots \wedge e_{r} \\
& +\sum_{j=2}^{r} \sum_{h, l=r+1}^{m-1} v_{h} \lambda_{l}^{j} e_{h} \wedge B \wedge e_{2} \wedge \cdots \wedge e_{j-1} \wedge e_{l} \wedge e_{j+1} \wedge \cdots \wedge e_{r} .
\end{aligned}
$$

From (136) we see that $w_{11}(s) \neq 0$.

Differentiating (137) with respect to $t_{j}(j=2, \ldots, r)$, with the aid of (138) we get

$$
\sum_{h=r+1}^{m-1}\left(\left\langle B^{\prime}, e_{j}^{\prime}\right\rangle v_{h}-v_{j}^{\prime} v_{h}-w_{11} \lambda_{h}^{j}\right) e_{h} \wedge B \wedge e_{2} \wedge \cdots \wedge e_{r}=\mathbf{0},
$$

which implies

$$
v_{h} \sum_{p=r+1}^{m-1} v_{p} \lambda_{p}^{j}-v_{j}^{\prime} v_{h}-w_{11} \lambda_{h}^{j}=0
$$

as the coefficient of the vector $e_{h} \wedge B \wedge e_{2} \wedge \cdots \wedge e_{r}$ for all $j=2, \ldots, r$ and $h=r+1, \ldots, m-1$.

If $v_{h}=0$ for all $h,(134)$ implies that $B^{\prime}$ is tangent to $M$. With the help of Lemma 8 and Theorem 3 , we can see that $M$ is minimal and hence the Gauss map $G$ of $M$ is harmonic. In this case, $G$ can be chosen as the constant vector $-\mathbf{C}$. That is, $M$ is part of a Lorentzian $(r+1)$-plane in $\mathbb{L}^{m}$.

Now, we suppose that $v_{h} \neq 0$ for some $h \in\{r+1, \ldots, m-1\}$. If we put

$$
e_{h}^{\prime}=v_{h} A-u_{h} B-\sum_{j=2}^{r} \lambda_{h}^{j} e_{j}
$$

in the same manner as (15) by virtue of Lemma 1, differentiating (138) with respect to $s$ provides 


$$
\begin{aligned}
w_{11}^{\prime} \mathbf{C}= & \sum_{h=r+1}^{m-1}\left\{v_{h}^{\prime \prime}+\left(\sum_{j=2}^{r} v_{j}^{2}\right) u_{h}-\sum_{j=2}^{r} v_{j} u_{j} v_{h}+\sum_{j=2}^{r} \sum_{p=r+1}^{m-1} v_{p} \lambda_{p}^{j} \lambda_{h}^{j}-\sum_{j=2}^{r} \sum_{p=r+1}^{m-1}\left(\lambda_{p}^{j}\right)^{2} v_{h}\right\} \\
& +\left(\sum_{p=r+1}^{m-1} v_{p} v_{p}^{\prime}-2 \sum_{j=2}^{r} v_{j} v_{j}^{\prime}+\sum_{j=2}^{r} \sum_{p=r+1}^{m-1} v_{j} \lambda_{p}^{j} v_{p}\right) A \wedge B \wedge e_{r} \wedge \cdots \wedge e_{r} \\
& +\sum_{h, p=r+1}^{m-1}\left(v_{h}^{\prime} v_{p}-\sum_{j=2}^{r} v_{j} v_{h} \lambda_{p}^{j}\right) e_{h} \wedge e_{p} \wedge e_{2} \wedge \cdots \wedge e_{r} \\
& -\sum_{j=2}^{r} \sum_{h=r+1}^{m-1}\left\{v_{j} v_{h}^{\prime}+\left(\sum_{k=1}^{r} v_{k}^{2}\right) \lambda_{h}^{j}+\left(v_{j} v_{h}\right)^{\prime}-\left(\sum_{p=r+1}^{m-1} v_{p}^{2}\right) \lambda_{h}^{j}+\sum_{p=r+1}^{m-1} v_{p} \lambda_{p}^{j} v_{h}\right\} \\
& A \wedge B \wedge e_{2} \wedge \cdots \wedge e_{j-1} \wedge e_{h} \wedge e_{j+1} \wedge \cdots \wedge e_{r} \\
& +\sum_{j=2}^{r} \sum_{h, p=r+1}^{m-1}\left\{v_{h}^{\prime} \lambda_{p}^{j}-v_{j} v_{h} u_{p}+\left(v_{h} \lambda_{p}^{j}\right)^{\prime}\right\} e_{h} \wedge B \wedge e_{2} \wedge \cdots \wedge e_{j-1} \wedge e_{p} \wedge \cdots \wedge e_{r} .
\end{aligned}
$$

Comparing the coefficients of the vectors in (138) and (141), we obtain the following four equations:

$$
\begin{gathered}
\frac{w_{11}^{\prime}}{w_{11}} v_{h}^{\prime}=v_{h}^{\prime \prime}+\left(\sum_{j=2}^{r} v_{j}^{2}\right) u_{h}-\sum_{j=2}^{r} v_{j} u_{j} v_{h}+\sum_{j=2}^{r} \sum_{p=r+1}^{m-1} v_{p} \lambda_{p}^{j} \lambda_{h}^{j}-\sum_{j=2}^{r} \sum_{p=r+1}^{m-1}\left(\lambda_{p}^{j}\right)^{2} v_{h}, \\
-\frac{w_{11}^{\prime}}{w_{11}}\left(\sum_{j=2}^{r} v_{j}^{2}\right)=\sum_{p=r+1}^{m-1} v_{p} v_{p}^{\prime}-2 \sum_{j=2}^{r} v_{j} v_{j}^{\prime}+\sum_{j=2}^{r} \sum_{p=r+1}^{m-1} v_{j} \lambda_{p}^{j} v_{p}, \\
v_{h}^{\prime} v_{p}-\sum_{j=2}^{r} v_{j} v_{h} \lambda_{p}^{j}-v_{p}^{\prime} v_{h}+\sum_{j=2}^{r} v_{j} v_{p} \lambda_{h}^{j}=0, \\
\frac{w_{11}^{\prime}}{w_{11}} v_{j} v_{h}=v_{j} v_{h}^{\prime}+\left(\sum_{k=2}^{r} v_{k}^{2}\right) \lambda_{h}^{j}+\left(v_{j} v_{h}\right)^{\prime}-\left(\sum_{p=r+1}^{m-1} v_{p}^{2}\right) \lambda_{h}^{j}+v_{h} \sum_{p=r+1}^{m-1} v_{p} \lambda_{p}^{j} .
\end{gathered}
$$

Substituting (139) into (143), we get

$$
-\frac{w_{11}^{\prime}}{w_{11}}\left(\sum_{j=2}^{r} v_{j}^{2}\right)=\sum_{p=r+1}^{m-1} v_{p} v_{p}^{\prime}-\sum_{j=2}^{r} v_{j} v_{j}^{\prime}+\frac{w_{11}}{v_{h}} \sum_{k=2}^{r} v_{k} \lambda_{h}^{k}
$$

for some $h$ with $v_{h} \neq 0$. Putting (146) into (144) gives

$$
v_{p} v_{h}^{\prime}=v_{h} v_{p}^{\prime}
$$

which implies

$$
v_{h}(s)=c_{p}^{h} v_{p}(s)
$$

for some constant $c_{p}^{h}$. Therefore, we can put

$$
v_{h}(s)=c_{h} v_{r+1}(s)
$$

for some constants $c_{h}$ and $h=r+1, \ldots, m-1$.

Recall that Equation (139) is valid for all $h=r+1, \ldots, m-1$. By replacing $h$ with $r+1, \ldots, m-1$, respectively, and comparing equations obtained in such a way, with the help of (147) we can get 


$$
\lambda_{h}^{j}(s)=c_{h} \lambda_{r+1}^{j}(s)
$$

for all $j=2, \ldots, r$ and for all $h=r+1, \ldots, m-1$. By virtue of (147) and (148), Equation (142) is simplified as

$$
\frac{w_{11}^{\prime}}{w_{11}} v_{h}^{\prime}=v_{h}^{\prime \prime}+\left(\sum_{j=2}^{r} v_{j}^{2}\right) u_{h}-\sum_{j=2}^{r} v_{j} u_{j} v_{h}
$$

for all $h=r+1, \ldots, m-1$. Putting (147) into (149) and repeating the method to get (148), we have

$$
u_{h}(s)=c_{h} u_{r+1}(s)
$$

for all $h=r+1, \ldots, m-1$. If we put (147) and (148) into (139), then we obtain

$$
v_{j}^{\prime} v_{r+1}=-\left(\sum_{k=2}^{r} v_{k}^{2}\right) \lambda_{r+1}^{j}
$$

because of $w_{11}=\sum_{j} v_{j}^{2}+\sum_{h} v_{h}^{2}$. Substituting (139) into (145) provides

$$
\frac{w_{11}^{\prime}}{w_{11}} v_{j} v_{r+1}=2\left\{\left(v_{j} v_{r+1}\right)^{\prime}+\left(\sum_{k=2}^{r} v_{k}^{2}\right) \lambda_{r+1}^{j}\right\},
$$

which yields

$$
v_{j}\left(\frac{w_{11}^{\prime}}{w_{11}} v_{r+1}-2 v_{r+1}^{\prime}\right)=0
$$

with the help of (151).

If $v_{j}=0,(138)$ implies that

$$
w_{11} \mathbf{C}=\sum_{h=r+1}^{m-1} v_{h}^{\prime} e_{h} \wedge B \wedge e_{2} \wedge \cdots \wedge e_{r}
$$

and hence

$$
w_{11}^{\prime} \mathbf{C}=\sum_{h=r+1}^{m-1} v_{h}^{\prime \prime} e_{h} \wedge B \wedge e_{2} \wedge \cdots \wedge e_{r}+\sum_{h=r+1}^{m-1} v_{h} v_{h}^{\prime} A \wedge B \wedge e_{2} \wedge \cdots \wedge e_{r} .
$$

Combining (153) and (154), we have

$$
\sum_{h=r+1}^{m-1} v_{h} v_{h}^{\prime}=0
$$

which means that the function $w_{11}=\sum_{h} v_{h}^{2}$ is constant. Since $w_{11}=v_{r+1}^{2} \sum_{h} c_{h}^{2}$, the function $v_{r+1}$ is constant, so are $v_{h}$ for all $h=r+1, \ldots, m-1$. In (153), we can see that $C$ is a zero vector because of $w_{11} \neq 0$, a contradiction. Therefore, from (152), we conclude that

$$
\frac{w_{11}^{\prime}}{w_{11}} v_{r+1}=2 v_{r+1}^{\prime}
$$

or, equivalently,

$$
w_{11}=d_{r+1} v_{r+1}^{2}
$$

for some positive constant $d_{r+1}$. Since $w_{11}=\sum_{j} v_{j}^{2}+\sum_{h} v_{h}^{2}=\sum_{j} v_{j}^{2}+v_{r+1}^{2} \sum_{h} c_{h}^{2}$, we see that

$$
\sum_{j=2}^{r} v_{j}^{2}=\left(d_{r+1}-\sum_{h=r+1}^{m-1} c_{h}^{2}\right) v_{r+1}^{2} .
$$


We now introduce another kind of generalized null scroll as follows:

For a null curve $\tilde{\alpha}(s)$ in $\mathbb{L}^{m}$, we consider a null frame $\left\{A(s), B(s)=e_{1}(s), e_{2}(s), \ldots, e_{m-1}(s)\right\}$ along $\tilde{\alpha}(s)$ satisfying

$$
\begin{array}{r}
\langle A(s), A(s)\rangle=\langle B(s), B(s)\rangle=\left\langle A(s), e_{i}(s)\right\rangle=\left\langle B(s), e_{i}(s)\right\rangle=0, \\
\langle A(s), B(s)\rangle=-1,\left\langle e_{i}(s), e_{j}(s)\right\rangle=\delta_{i j}, \quad \tilde{\alpha}^{\prime}(s)=A(s)
\end{array}
$$

for $i, j=2,3, \ldots, m-1$.

Let $X(s)$ be the matrix $\left(A(s) B(s) e_{2}(s) \cdots e_{m-1}(s)\right)$ consisting of column vectors of $A(s), B(s)$, $e_{2}(s), \ldots, e_{m-1}(s)$ with respect to the standard coordinate system in $\mathbb{L}^{m}$. Then we have

$$
X^{t}(s) E X(s)=T,
$$

where $X^{t}(s)$ denotes the transpose of $X(s), E=\operatorname{diag}(-1,1, \ldots, 1)$ and

$$
T=\left(\begin{array}{ccccc}
0 & -1 & 0 & \cdots & 0 \\
-1 & 0 & 0 & \cdots & 0 \\
0 & 0 & 1 & \cdots & 0 \\
\vdots & \vdots & \vdots & \ddots & \vdots \\
0 & 0 & 0 & \cdots & 1
\end{array}\right)
$$

Consider a system of ordinary differential equations

$$
X^{\prime}(s)=X(s) M(s)
$$

where

$$
M(s)=\left(\begin{array}{ccccccccc}
0 & 0 & v_{2} & \cdots & v_{r} & v_{r+1} & c_{r+2} v_{r+1} & \cdots & c_{m-1} v_{r+1} \\
0 & 0 & -u_{2} & \cdots & -u_{r} & -u_{r+1} & -c_{r+2} u_{r+1} & \cdots & -c_{m-1} u_{r+1} \\
-u_{2} & v_{2} & 0 & \cdots & 0 & -\lambda_{r+1}^{2} & -c_{r+2} \lambda_{r+1}^{2} & \cdots & -c_{m-1} \lambda_{r+1}^{2} \\
-u_{3} & v_{3} & 0 & \cdots & 0 & -\lambda_{r+1}^{3} & -c_{r+2} \lambda_{r+1}^{3} & \cdots & -c_{m-1} \lambda_{r+1}^{3} \\
\vdots & \vdots & \vdots & & \vdots & \vdots & \vdots & & \vdots \\
-u_{r} & v_{r} & 0 & \cdots & 0 & -\lambda_{r+1}^{r} & -c_{r+2} \lambda_{r+1}^{r} & \cdots & -c_{m-1} \lambda_{r+1}^{r} \\
-u_{r+1} & v_{r+1} & \lambda_{r+1}^{2} & \cdots & \lambda_{r+1}^{r} & 0 & 0 & \cdots & 0 \\
-c_{r+2} u_{r+1} & c_{r+2} v_{r+1} & c_{r+2} \lambda_{r+1}^{2} & \cdots & c_{r+2} \lambda_{r+1}^{r} & 0 & 0 & \cdots & 0 \\
\vdots & \vdots & \vdots & & \vdots & \vdots & \vdots & & \vdots \\
-c_{m-1} u_{r+1} & c_{m-1} v_{r+1} & c_{m-1} \lambda_{r+1}^{2} & \cdots & c_{m-1} \lambda_{r+1}^{r} & 0 & 0 & \cdots & 0
\end{array}\right),
$$

where $v_{i}(2 \leq i \leq r+1), u_{j}(2 \leq j \leq r+1)$ and $\lambda_{r+1}^{k}(2 \leq k \leq r, r+1 \leq b \leq m-1)$ are some smooth functions of $s$ and $c_{h}(r+1 \leq h \leq m-1)$ are constant satisfying

$$
\begin{array}{r}
\sum_{j=2}^{r} v_{j}^{2}=d v_{r+1}^{2}, \quad v_{j}^{\prime}=-d v_{r+1} \lambda_{r+1}^{j} \quad \text { and } \\
\frac{w_{11}^{\prime}}{w_{11}} v_{r+1}^{\prime}=v_{r+1}^{\prime \prime}+\left(\sum_{j=2}^{r} v_{j}^{2}\right) u_{r+1}-\sum_{j=2}^{r} v_{j} u_{j} v_{r+1}
\end{array}
$$

for some positive constant $d$. 
For a given initial condition $X(0)=\left(A(0) B(0) e_{2}(0) \cdots e_{m-1}(0)\right)$ satisfying $X^{t}(0) E X(0)=T$, there exists a unique solution to $X^{\prime}(s)=X(s) M(s)$ on the whole domain $I$ of $\tilde{\alpha}(s)$ containing 0 . Since $T$ is symmetric and MT is skew-symmetric, $\frac{d}{d s}\left(X^{t}(s) E X(s)\right)=0$ and hence we have

$$
X^{t}(s) E X(s)=T
$$

for all $s \in I$. Therefore, $A(s), B(s), e_{2}(s), \ldots, e_{m-1}(s)$ form a null frame along a null curve $\tilde{\alpha}(s)$ in $\mathbb{L}^{m}$ on $I$. Let $\alpha(s)=\int_{0}^{s} A(u) d u$.

We now give the following definition.

Definition 2. A generalized null scroll satisfying (157) parameterized by

$$
x\left(s, t_{1}, t_{2}, \cdots, t_{r}\right)=\alpha(s)+t_{1} B(s)+\sum_{i=2}^{r} t_{i} e_{i}(s) .
$$

is called the generalized B-scroll kind.

Remark 2. In the case of $m=3$ with $v_{2} \in \mathbb{R}$, a generalized B-scroll kind is a so-called B-scroll.

Therefore, we can see that the parametrization of a generalized null scroll $M$ with a pointwise 1-type Gauss map of the second kind can be given by (159). Furthermore, by combining the first two equations of (158), we can see that these ruled submanifolds satisfy

$$
v_{r+1}^{\prime}=-\sum_{j=2}^{r} v_{j} \lambda_{r+1}^{j}
$$

Conversely, for a generalized $B$-scroll kind $M$ parameterized by (159), by computation, $\Delta G$ can be expressed as

$$
\Delta G=\frac{2 w_{11}}{Q^{2}}(G+\mathbf{C}),
$$

where $\mathbf{C}$ is the constant vector given by

$$
\begin{aligned}
\mathbf{C}=\frac{1}{w_{11}}\{ & \sum_{h=r+1}^{m-1} c_{h} v_{r+1}^{\prime} e_{h} \wedge B \wedge e_{2} \wedge \cdots \wedge e_{r}-\sum_{j=2}^{r} v_{j}^{2} A \wedge B \wedge e_{2} \wedge \cdots \wedge e_{r} \\
& \left.+\sum_{j=2}^{r} \sum_{h=r+1}^{m-1} v_{j} c_{h} v_{r+1} e_{h} \wedge B \wedge e_{2} \wedge \cdots \wedge e_{j-1} \wedge A \wedge e_{j+1} \wedge \cdots \wedge e_{r}\right\} .
\end{aligned}
$$

It means that the Gauss map $G$ of $M$ is of pointwise 1-type of the second kind.

Case 6. $f_{t_{1}} \neq 0$.

In this case, the open subset $W=\left\{p \in M \mid f_{t_{1}}(p) \neq 0\right\}$ is non-empty. Comparing the vectors composing the constant vector $\mathbf{C}$ of (133) and (135), by the orthogonality of them, we get

$$
v_{j} v_{h}=0 \quad \text { and } \quad v_{h} \lambda_{p}^{j}-v_{p} \lambda_{h}^{j}=0
$$

on $W$ for all $j=2, \ldots, r$ and $h, p=r+1, \ldots, m-1$.

If $v_{h}=0$ for all $h=r+1, \ldots, m-1$, we obtain the result that the open subset $W$ of $M$ is part of a Lorentzian $(r+1)$-plane by Lemma 8 and Theorem 3 .

If $v_{h} \neq 0$ for some $h \in\{r+1, \ldots, m-1\}$, then $v_{j}=0$ and $Q=1$ for all $j=2, \ldots, r$. Then, Equation (133) is simplified as 


$$
\begin{aligned}
& 2 \sum_{h=r+1}^{m-1}\left\{\left(\sum_{i=1}^{r}\left\langle B^{\prime}, e_{i}^{\prime}\right\rangle v_{h} t_{i}+v_{h}^{\prime}\right)\right\} e_{h} \wedge B \wedge e_{2} \wedge \cdots \wedge e_{r} \\
& +2 \sum_{h=r+1}^{m-1} v_{h}^{2} A \wedge B \wedge e_{2} \wedge \cdots \wedge e_{r} \\
= & f A \wedge B \wedge e_{2} \wedge \cdots \wedge e_{r}+f \sum_{h=r+1}^{m-1}\left(t_{1} v_{h}+\sum_{j=2}^{r} \lambda_{h}^{j} t_{j}\right) e_{h} \wedge B \wedge e_{2} \wedge \cdots \wedge e_{r}+f \mathbf{C},
\end{aligned}
$$

or,

$$
\begin{aligned}
\mathbf{C}= & \frac{1}{f}\left\{2 \sum_{h=r+1}^{m-1}\left\{\left(\sum_{i=1}^{r}\left\langle B^{\prime}, e_{i}^{\prime}\right\rangle v_{h} t_{i}+v_{h}^{\prime}\right)\right\} e_{h} \wedge B \wedge e_{2} \wedge \cdots \wedge e_{r}\right. \\
& \left.+\left(2 \sum_{h=r+1}^{m-1} v_{h}^{2}-f\right) A \wedge B \wedge e_{2} \wedge \cdots \wedge e_{r}\right\} \\
& -\sum_{h=r+1}^{m-1}\left(t_{1} v_{h}+\sum_{j=2}^{r} \lambda_{h}^{j} t_{j}\right) e_{h} \wedge B \wedge e_{2} \wedge \cdots \wedge e_{r}
\end{aligned}
$$

on $W$. By differentiating (160) with respect to $t_{1}$ and using (161), we can obtain

$$
\frac{2 f_{t_{1}}}{f} \sum_{h=r+1}^{m-1} v_{h}^{2}=0
$$

as the coefficients of $A \wedge B \wedge e_{2} \wedge \cdots \wedge e_{r}$. Since $f_{t_{1}} \neq 0$ on $W$, we have $\sum_{h} v_{h}^{2}=0$, a contradiction to $v_{h} \neq 0$ for some $h$.

Therefore, we can conclude that if the open set $W$ is non-empty, then the functions $v_{h}$ are identically zero on $W$ for all $h=r+1, \ldots, m-1$, and hence we see that $W$ is an open part of a Lorentzian plane in $\mathbb{L}^{m}$. By continuity, $M$ is a Lorentzian $(r+1)$-plane.

Therefore, we have

Theorem 4. Let $M$ be a generalized null scroll in the Minkowski m-space $\mathbb{L}^{m}$. Then, $M$ has a pointwise 1-type Gauss map of the second kind if and only if $M$ is part of a Lorentzian $(r+1)$-plane in $\mathbb{L}^{m}$ or a generalized B-scroll kind.

In particular, by straightforward computation, we have

Corollary 1. Let $M$ be a null scroll in the Minkowski 3-space $\mathbb{L}^{3}$. Then, M has a pointwise 1-type Gauss map of the second kind if and only if $M$ is part of a time-like plane or a flat B-scroll.

Author Contributions: Y.H.K. gave the idea to start the research on Gauss Map and its applications on ruled submanifolds in Minkowski Space, computed the last section and polish the draft. S.M.J. devoted to compute the details.

Funding: The second named author was supported by Kyungpook National University Bokhyeon Research Fund, 2017.

Acknowledgments: Authors would like to express their appreciation to the referees for their comments and valuable suggestions to improve the paper.

Conflicts of Interest: The authors declare no conflict of interest.

\section{References}

1. Chen, B.-Y. Total Mean Curvature and Submanifolds of Finite Type, 2nd ed.; World Scientific: Singapore, 2015.

2. Baikoussis, C. Ruled submanifolds with finite type Gauss map. J. Geom. 1994, 49, 42-45. [CrossRef] 
3. Baikoussis, C.; Chen, B.-Y.; Verstraelen, L. Ruled surfaces and tubes with finite type Gauss map. Tokyo J. Math. 1993, 16, 341-349. [CrossRef]

4. Chen, B.-Y. A report on submanifolds of finite type. Soochow J. Math. 1996, 22, 117-337.

5. Chen, B.-Y.; Dillen, F.; Verstraelen, L.; Vrancken, L. Ruled surfaces of finite-type. Bull. Aust. Math. Soc. 1990, 42, 447-453. [CrossRef]

6. Dillen, F. Ruled submanifolds of finite type. Proc. Am. Math. Soc. 1992, 114, 795-798. [CrossRef]

7. Kim, D.-S.; Kim, Y.H. Some classification results on finite-type ruled submanifolds in a Lorentz-Minkowski space. Taiwan. J. Math. 2012, 16, 1475-1488. [CrossRef]

8. Kim, D.-S.; Kim, Y.H. Minimal ruled submanifolds in Minkowski space $\mathbb{L}^{m}$. J. Geom. Phys. 2012, 62, 1893-1902. [CrossRef]

9. Kim, D.-S.; Kim, Y.H.; Jung, S.M. Ruled submanifolds with harmonic Gauss map. Taiwan. J. Math. 2014, 18, 53-76. [CrossRef]

10. Kim, D.-S.; Kim, Y.H.; Jung, S.M. Some classifications of ruled submanifolds in Minkowski space and their Gauss map. Taiwan. J. Math. 2014, 18, 1021-1040. [CrossRef]

11. Kim, D.-S.; Kim, Y.H.; Yoon, D.W. Finite-type ruled surfaces in Lorentz-Minkowski space. Taiwan. J. Math. 2007, 11, 1-13. [CrossRef]

12. Kim, D.-S.; Kim, Y.H.; Yoon, D.W. Extended B-scrolls and their Gauss maps. Indian J. Pure Appl. Math. 2002, 33, 1031-1040.

13. Kim, D.-S.; Kim, Y.H.; Yoon, D.W. Characterization of generalized B-scrolls and cylinders over finite-type curves. Indian J. Pure Appl. Math. 2003, 33, 1523-1532.

14. Kim, Y.H.; Yoon, D.W. Classification of ruled surfaces in Minkowski 3-spaces. J. Geom. Phys. 2004, 49, 89-100. [CrossRef]

15. Kim, Y.H.; Yoon, D.W. On the Gauss map of ruled surfaces in Minkowski space. Rocky Mt. J. Math. 2005, 35, 1555-1581. [CrossRef]

16. Choi, M.; Kim, Y.H. Characterization of the helicoid as ruled surfaces with pointwise 1-type Gauss map. Bull. Korean Math. Soc. 2001, 38, 753-761.

17. Choi, M.; Kim, D.-S.; Kim, Y.H.; Yoon, D.W. Circular cone and its Gauss map. Colloq. Math. 2012, 129, 203-210. [CrossRef]

18. Chen, B.-Y.; Choi, M.; Kim, Y.H. Surfaces of revolution with pointwise 1-type Gauss map. J. Korean Math. Soc. 2005, 42, 447-455. [CrossRef]

19. Jung, S.M.; Kim, D.-S.; Kim, Y.H.; Yoon, D.W. Gauss maps of ruled submanifolds and applications I. J. Korean Math. Soc. 2016, 53, 1309-1330. [CrossRef]

20. Kim, D.-S.; Kim, Y.H.; Jung, S.M.; Yoon, D.W. Gauss maps of ruled submanifolds and applications $I I$. Taiwan. J. Math. 2016, 20, 227-242. [CrossRef]

21. Choi, M.; Kim, Y.H.; Yoon, D.W. Classification of ruled surfaces with pointwise 1-type Gauss map. Taiwan. J. Math. 2010, 14, 1297-1308. [CrossRef]

22. Choi, M.; Kim, Y.H.; Yoon, D.W. Classification of ruled surfaces with pointwise 1-type Gauss map in Minkowski 3-space. Taiwan. J. Math. 2011, 15, 1141-1161. [CrossRef]

23. Choi, M.; Kim, D.-S.; Kim, Y.H. Helicoidal surfaces with pointwise 1-type Gauss map. J. Korean Math. Soc. 2009, 46, 215-233. [CrossRef]

24. Kim, Y.H.; Yoon, D.W. Ruled surfaces with pointwise 1-type Gauss maps. J. Geom. Phys. 2000, 34, $191-205$. [CrossRef]

25. Karacan, M.K.; Yuksel, N.; Ikiz, H. On ruled surface in 3-dimenional almost contact metric manifold. Int. J. Geom. Methods Mod. Phys. 2017, 14. [CrossRef]

26. Maeda, S.; Tanabe, H. Sectional curvatures of ruled real hypersurfaces in complex hyperbolic spaces. Differ. Geom. Appl. 2017, 51, 1-8. [CrossRef]

27. Vilcu, G.E. On generic submanifolds of manifolds endowed with metric mixed 3-structures. Commun. Contemp. Math. 2016, 18, 1550081. [CrossRef]

28. Miroslava, A. Ruled three-dimensional CR submanifolds of the sphere. Publ. Inst. Math. 2017, 101, $25-35$. 
29. Jung, S.M.; Kim, D.-S.; Kim, Y.H. Minimal ruled submanifolds associated with Gauss map. Taiwan. J. Math. 2018, 22, 567-605. [CrossRef]

30. Barbosa, J.M.; Dajczer, M.; Jorge, I.P. Minimal ruled submanifolds in spaces of constant curvature. Indiana Univ. Math. J. 1984, 33, 531-547. [CrossRef]

(C) 2018 by the authors. Licensee MDPI, Basel, Switzerland. This article is an open access article distributed under the terms and conditions of the Creative Commons Attribution (CC BY) license (http:/ / creativecommons.org/licenses/by/4.0/). 